\title{
Pseudo-impulsive solutions of the forward-speed diffraction problem using a high- order finite-difference method
}

\author{
Amini-Afshar, Mostafa; Bingham, Harry B.
}

Published in:

Applied Ocean Research

Link to article, DOI:

10.1016/j.apor.2018.08.017

Publication date:

2018

Document Version

Peer reviewed version

Link back to DTU Orbit

Citation (APA):

Amini-Afshar, M., \& Bingham, H. B. (2018). Pseudo-impulsive solutions of the forward-speed diffraction problem using a high-order finite-difference method. Applied Ocean Research, 80, 197-219.

https://doi.org/10.1016/j.apor.2018.08.017

\section{General rights}

Copyright and moral rights for the publications made accessible in the public portal are retained by the authors and/or other copyright owners and it is a condition of accessing publications that users recognise and abide by the legal requirements associated with these rights.

- Users may download and print one copy of any publication from the public portal for the purpose of private study or research.

- You may not further distribute the material or use it for any profit-making activity or commercial gain

- You may freely distribute the URL identifying the publication in the public portal

If you believe that this document breaches copyright please contact us providing details, and we will remove access to the work immediately and investigate your claim. 


\title{
Pseudo-impulsive solutions of the forward-speed diffraction problem using a high-order finite-difference method
}

\author{
Mostafa Amini-Afshar and Harry B. Bingham \\ Technical University of Denmark \\ Department of Mechanical Engineering \\ Section for Fluid Mechanics, Coastal \& Maritime Engineering \\ maaf@mek.dtu.dk, hbb@mek.dtu.dk
}

\begin{abstract}
This paper considers pseudo-impulsive numerical solutions to the forward-speed diffraction problem, as derived from classical linearized potential flow theory. Both head- and following-seas cases are treated. Fourth-order finite-difference approximations are applied on overlapping, boundary-fitted grids to obtain solutions using both the Neumann-Kelvin and the double-body flow linearizations of the problem. A method for computing the pseudo-impulsive incident wave forcing in finite water depth using the Fast Fourier Transform (FFT) is presented. The pseudo-impulsive scattering solution is then Fourier transformed into the frequency domain to obtain the wave excitation forces and the body motion response. The calculations are validated against reference solutions for a submerged circular cylinder and a submerged sphere. Calculations are also made for a modern bulk carrier, showing good agreement with experimental measurements.
\end{abstract}

\section{Keywords}

Wave forces, Finite difference, Overlapping grids, Following seas, Diffraction problem, Forward speed

\section{Introduction}

Accurately predicting the wave-induced loading and response of sailing ships is important to ensure their safety and reliability. While high-fidelity numerical methods based on solving the NavierStokes equations (CFD) are now feasible for simulating short-term individual events, they are still too computationally expensive for routine analysis and preliminary design optimization, see for example $[1,2,3,4,5]$ and $[6]$. Thus, methods based on the assumptions of a linearized potential flow are still heavily used to map out the complete response spectrum, guide the initial design process and set up extreme loading scenarios for more refined CFD analysis.

The most efficient linearized potential flow solutions to this problem are obtained using twodimensional (2D) strip-type methods, for example based on [7] or [8]. Despite the theoretical weaknesses inherent to these methods, they generally produce excellent results for integrated quantities like global loading and motion response, although detailed local quantities are not available. Threedimensional (3D) methods are also widely used, most commonly based on the boundary element method (BEM) and the free-space (Rankine) Green function e.g. [9, 10, 11, 12, 13, 14]. A smaller number of time-domain solutions using the free-surface Green function have also been developed, for example [15], [16], [17], [18]. Finite element models for wave structure interaction problems can also be found, for example [19] and [20]. Two well-known cases of the use of finite difference method for the ship wave resistance problem are [21] and [22]. 
Motivated by the difficulties faced by BEM methods in obtaining a linear scaling of the solution effort with increasing resolution, we have been developing a high-order finite difference framework for nonlinear water wave simulation and wave-structure interaction, see for example $[23,24,25,26,27$, $28,29]$ and [30]. While the total number of unknowns required here is typically an order of magnitude larger than that required by BEM methods, the resulting system matrix is sparse, leading to a linear scaling of the solution effort, as demonstrated for example by [26]. This is especially attractive for computing second- and higher-order wave forces (for example added resistance [31]), where convergence of the solution typically requires high resolution.

In this paper, we present our solution to the forward-speed diffraction problem as implemented in the above described high-order finite-difference framework. Either the Neumann-Kelvin or the doublebody flow linearization may be adopted. Inspired by the work of [15], solutions are obtained in the time-domain using a pseudo-impulsive incident wave which is tuned to include only a limited range of frequencies. The pseudo-impulsive incident wave potential and its derivatives can be computed from elementary functions in infinite water depth, but in finite water depth no simple closed-form representation is available. In this case, we compute the wave kinematics using Fast Fourier Transforms, a fast and accurate method which has not yet appeared in the literature (to our knowledge).

A method of lines approach [32] is applied to obtain the discrete solution, with the explicit fourthorder Runge-Kutta scheme chosen for the time-integration. For the Laplace problem, fourth-order spatial finite difference schemes are developed on overlapping, boundary-fitted grids, resulting in a consistently fourth-order accurate solution, as demonstrated by [26]. Frequency-domain quantities are obtained by taking the Fourier transform of the time-domain results, and forces are computed by integrating the pressure over the surface of the body (using at least fourth-order accurate integration schemes).

The implementation supports waves incident from both ahead of the beam $(\pi / 2 \leq \beta \leq 3 \pi / 2$, head seas), and from abaft the beam $(0 \leq \beta<\pi / 2$ or $3 \pi / 2<\beta \leq 2 \pi$, following seas). Since the solution is obtained in a moving frame of reference fixed to the mean position of the ship, the following seas problem is complicated by the non-unique relationship between encounter frequency $\omega_{e}$ and wave frequency $\omega_{0}$. Following, for example [17] and [33], we solve three distinct pseudo-impulsive diffraction problems in this case. Port/starboard symmetry of the ship geometry can also be exploited to solve on only half of the physical domain.

Validation results are presented using both semi-analytical solutions and experimental measurements. First the forward-speed wave excitation forces on both a submerged circular cylinder and a submerged sphere are shown to converge towards the corresponding semi-analytical results. Then a modern bulk carrier is analyzed in both head and following seas, and both wave forces and body motions are shown to compare very well with experimental measurements and other numerical calculations. Finally we note that the model described here, OceanWave3D-Seakeeping [34], is available as an open-source code.

\section{Formulation of the problem}

The details of the classic mathematical formulation for ship motions including the diffraction problem, can be found for example in [35]. In this section, we include a brief review of the theory and illustrate the details of our solution. The focus here is on the diffraction problem, while the details of our solution to the radiation problem can be found in [26].

A coordinate system $O-x y z$ is considered which is attached to the mean position of the vessel and moves steadily with the same forward speed $U$. The incident wave makes an angle $\beta$ with the positive $\mathrm{x}$-axis, and is scattered by the moving vessel which has a steady forward speed $U$. See also Figure 1. Note that except for the steady forward motion, the vessel is assumed to be stationary in the diffraction problem. For the radiation problem, the body has (at least) six degrees of freedom denoted by: surge $\xi_{1}$, sway $\xi_{2}$, heave $\xi_{3}$, roll $\xi_{4}$, pitch $\xi_{5}$ and yaw $\xi_{6}$, which are also shown in Figure 1 . 


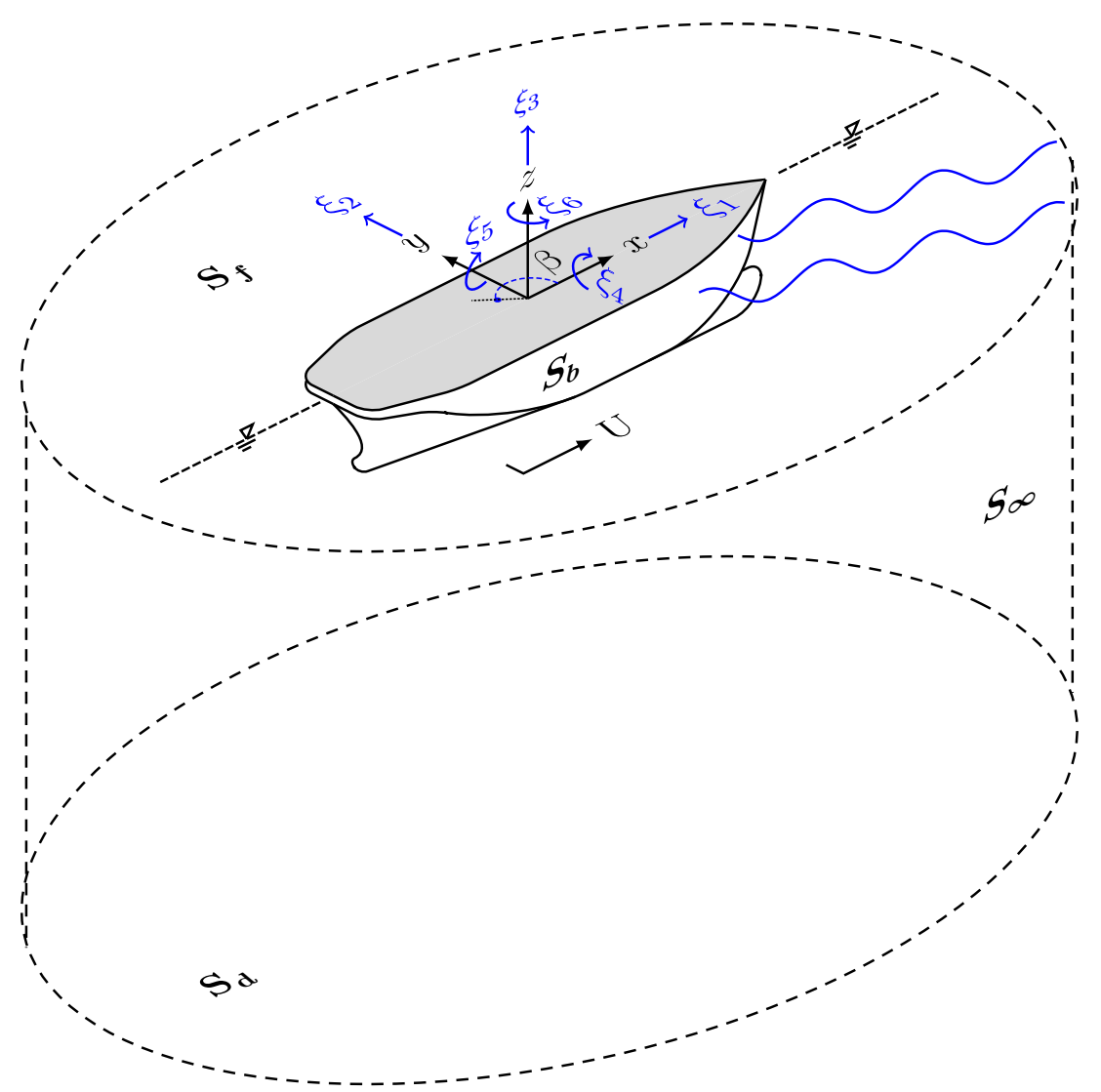

Figure 1: Sketch of the moving body and the flow domains.

\section{$2.1 \quad$ Governing equations}

The flow domain is bounded by the free surface $S_{f}$, the surface of the body $S_{b}$, the sea bed $S_{d}$ and the far-field truncation boundary $S_{\infty}$ which is required to limit the extent of the computational domain. Here $S_{f}$ and $S_{b}$ are the mean undisturbed free- and body-surfaces respectively. Assuming potential flow theory, all components of the flow velocity are defined by a velocity potential $\phi_{s}(x, y, z, t)$. This potential describes the flow due to the unknown scattered waves. The continuity condition can be specified by the Laplace equation as follows:

$$
\nabla^{2} \phi_{s}=\frac{\partial^{2} \phi_{s}}{\partial x^{2}}+\frac{\partial^{2} \phi_{s}}{\partial y^{2}}+\frac{\partial^{2} \phi_{s}}{\partial z^{2}}=0 .
$$

Note that the total velocity potential in the moving frame of reference can be decomposed as follows:

$$
\bar{\phi}_{s}=-U x+\phi_{b}^{\prime}+\phi_{s} .
$$

The base flow is shown here by $\phi_{b}^{\prime}$, and for the Neumann-Kelvin linearization $\phi_{b}^{\prime}=0$. For the case of the double-body linearization, the base flow can be obtained by solving the following steady-flow problem $\left(\phi_{b}^{\prime}=\phi_{d b}\right)$ :

$$
\begin{array}{rlrl}
\nabla^{2} \phi_{d b} & =0, & \\
\frac{\partial \phi_{d b}}{\partial n}=\boldsymbol{W} \cdot \boldsymbol{n} & & \text { on } S_{b}, \\
\frac{\partial \phi_{d b}}{\partial z} & =0 & & \text { on } z=0, \\
\nabla \phi_{d b} & \rightarrow 0 & & \text { in the far field }\left(S_{\infty}\right),
\end{array}
$$


where $\boldsymbol{W}=(U, 0,0)$ and $\boldsymbol{n}$ is the unit normal vector to the body surface, directed into the body (out of the fluid). This boundary value problem describes an infinite-domain potential flow around a combination of the body and its mirror image with respect to the $z=0$ plane.

\subsection{Boundary conditions}

The free surface $S_{f}$ is subject to the linear dynamic and kinematic conditions as follows:

$$
\begin{array}{rlrl}
\frac{\partial \phi_{s}}{\partial t} & =-g \zeta+U \frac{\partial \phi_{s}}{\partial x}-\nabla \phi_{b}^{\prime} \cdot \nabla \phi_{s} & \text { at } z=0 \\
\frac{\partial \zeta}{\partial t}=\frac{\partial \phi_{s}}{\partial z}+U \frac{\partial \zeta}{\partial x}-\nabla \phi_{b}^{\prime} \cdot \nabla \zeta+\zeta \frac{\partial^{2} \phi_{b}^{\prime}}{\partial z^{2}} & \text { at } z=0 .
\end{array}
$$

Here $g$ is the acceleration due to gravity and $\zeta$ defines the free-surface elevation of the scattered waves. At the sea bed $S_{d}$ and the far-field truncation boundary $S_{\infty}$ the following no-flux condition is applied:

$$
\boldsymbol{n} \cdot \nabla \phi_{s}=0
$$

At the surface of the body $S_{b}$ the similar no-flux condition is applied to the flow field comprised of the incident wave $\phi_{0}(x, y, z, t)$ and the scattered wave $\phi_{s}(x, y, z, t)$ :

$$
\boldsymbol{n} \cdot \nabla\left(\phi_{0}+\phi_{s}\right)=0 \quad \Longrightarrow \quad \boldsymbol{n} \cdot \nabla \phi_{s}=-\boldsymbol{n} \cdot \nabla \phi_{0} .
$$

The numerical solution to this initial boundary value problem is described in $\S 3$.

\subsection{Implementation of the body boundary condition}

In order to obtain the scattering velocity potential, it is required to know the body boundary condition in the time domain. To this end, first the velocity potential of a linear monochromatic incident wave is considered, which can be defined everywhere in the constant-depth domain by:

$$
\phi_{0}(\mathbf{r}, t)=\operatorname{Re}\left\{\frac{\mathrm{i} A g}{\omega_{0}} \frac{\cosh k(z+h)}{\cosh k h} e^{-\mathrm{i} k \alpha} e^{\mathrm{i} \omega_{e} t}\right\},
$$

in which $\mathbf{r}=(x, y, z)$ is the position vector, and $A$ is the amplitude of the incident wave, which without loss of generality is assumed to be unity. Here $\omega_{0}$ is the wave frequency and $\omega_{e}$ is the encounter frequency, and they are related through:

$$
\omega_{e}=\omega_{0}-k U \cos \beta,
$$

where $k=2 \pi / \lambda$ is the wave number defined for the wave with length $\lambda$. The heading angle is $\beta$ and is measured with respect to the positive $x$ axis. The water depth is given by $h$, and $\alpha$ is a phase function defined as:

$$
\alpha=x \cos \beta+y \sin \beta .
$$

Note that with the above definition, head-seas and following-seas conditions are given by $\beta=\pi$ and $\beta=0$ respectively. Moreover the incident wave elevation will be:

$$
\zeta_{0}(\mathbf{r}, t)=\operatorname{Re}\left\{e^{-\mathrm{i} k \alpha} e^{\mathrm{i} \omega_{e} t}\right\} .
$$

If the interest was to find the scattering velocity potential for a single incident wave with a specific frequency, then (2.11) would be employed to calculate the body boundary condition in the time domain which is required by (2.10). Making use of linear superposition however, it is much more efficient to consider a wider range of frequencies in a single time-domain calculation. 
Traditionally this strategy has been based upon introducing an impulse response function denoted by $\boldsymbol{K}(\mathbf{r}, t-\tau)$. If this impulse response function is known, then the velocities $\nabla \tilde{\phi}_{0}(\mathbf{r}, t)$ for any arbitrary incident wave elevation $\tilde{\zeta}_{0}(\tau)$ can be obtained by convolution [33]:

$$
\nabla \tilde{\phi}_{0}(\mathbf{r}, t)=\int_{-\infty}^{\infty} \boldsymbol{K}(\mathbf{r}, t-\tau) \tilde{\zeta}_{0}(\tau) d \tau
$$

This function defines the gradient of the velocity potential (flow velocity at position $\mathbf{r}$ and time $t$ ), due to an impulsive incident wave located at the origin of the coordinate system. This definition has been motivated by the fact that theoretically the impulse function in the time domain is equivalent to a constant-valued function over an infinite number of frequencies in the frequency domain. The wave velocities and the wave elevation are known in the case of a linear monochromatic incident wave, (2.11) and (2.14). These can be used to find the unknown impulse response function $\boldsymbol{K}(\mathbf{r}, t-\tau)$ as follows:

$$
\nabla \tilde{\phi}_{0}(\mathbf{r}, t)=\nabla \phi_{0}(\mathbf{r}, t)=\int_{-\infty}^{\infty} \boldsymbol{K}(\mathbf{r}, t-\tau) e^{\mathrm{i} \omega_{e} \tau} d \tau=e^{\mathrm{i} \omega_{e} t} \int_{-\infty}^{\infty} \boldsymbol{K}(\mathbf{r}, \tau) e^{-\mathrm{i} \omega_{e} \tau} d \tau
$$

Note that the change of variable $t-\tau=t^{\prime}$ has been used here. The wave velocities are already known from (2.11) and can be inserted which leads to:

$$
\nabla \phi_{0}(\mathbf{r}, t)=\operatorname{Re}\left\{\boldsymbol{\Psi} e^{-\mathrm{i} k \alpha} e^{\mathrm{i} \omega_{e} t}\right\}=\operatorname{Re}\left\{e^{\mathrm{i} \omega_{e} t} \int_{-\infty}^{\infty} \boldsymbol{K}(\mathbf{r}, \tau) e^{-\mathrm{i} \omega_{e} \tau} d \tau\right\}
$$

in which:

$$
\boldsymbol{\Psi}=\left(\begin{array}{c}
\frac{g k}{\omega_{0}} \frac{\cosh k(z+h)}{\cosh k h} \cos \beta \\
\frac{g k}{\omega_{0}} \frac{\cosh k(z+h)}{\cosh k h} \sin \beta \\
\mathrm{i} \frac{g k}{\omega_{0}} \frac{\sinh k(z+h)}{\cosh k h}
\end{array}\right) .
$$

As the two sinusoids are equal in (2.17), their corresponding complex phasor should be the same, which means that:

$$
\boldsymbol{\Psi} e^{-\mathrm{i} k \alpha}=\int_{-\infty}^{\infty} \boldsymbol{K}(\mathbf{r}, \tau) e^{-\mathrm{i} \omega_{e} \tau} d \tau
$$

Finally by applying the inverse Fourier transform to (2.19), the impulse response function can be obtained as follows:

$$
\boldsymbol{K}(\mathbf{r}, t)=\mathcal{F}^{-1}\left\{\boldsymbol{\Psi} e^{-i k \alpha}\right\}=\frac{1}{2 \pi} \int_{-\infty}^{\infty} \boldsymbol{\Psi} e^{-\mathrm{i} k \alpha} e^{\mathrm{i} \omega_{e} t} d \omega_{e}
$$

Here the Fourier transform pair for the function $\phi$ in the time domain and the function $\hat{\phi}$ in the frequency domain, based on the angular encounter frequency $\omega_{e}$ is defined by:

$$
\begin{gathered}
\mathcal{F}\{\phi(t)\}=\hat{\phi}\left(\omega_{e}\right)=\int_{-\infty}^{\infty} \phi(t) e^{-\mathrm{i} \omega_{e} t} d t \\
\mathcal{F}^{-1}\left\{\hat{\phi}\left(\omega_{e}\right)\right\}=\phi(t)=\frac{1}{2 \pi} \int_{-\infty}^{\infty} \hat{\phi}\left(\omega_{e}\right) e^{\mathrm{i} \omega_{e} t} d \omega_{e} .
\end{gathered}
$$

Note also that the analytical solution to (2.20) is given by [33] for the deep-water case. The well-known approach in the literature, is the application of the impulse response function calculated from (2.20) to build the body boundary condition as:

$$
\mathbf{n} \cdot \nabla \phi_{s}=-\mathbf{n} \cdot \boldsymbol{K}(\mathbf{r}, t) .
$$


Based on this boundary condition, the time-domain solution can be calculated for the scattering velocity potentials due to an impulsive incident wave. If this impulsive solution to $\phi_{s}$ is inserted in to the Bernoulli equation, then the well-known diffraction impulse function will be obtained. The Fourier transform of this impulse response function gives the wave excitation forces in the frequency domain. More details about the diffraction and the radiation impulse response function can be found for example in [16].

\subsection{Pseudo-impulsive wave elevation}

As first suggested by [33], the solution can also be expressed in terms of a pseudo-impulsive incident wave. The temporal variation of the amplitude of this pseudo-impulsive wave can be defined for example by a Gaussian function:

$$
\zeta_{0}(t)=e^{-2 \pi^{2} s^{2} t^{2}},
$$

where the parameter $s$ controls the "width" of the function and can be used to limit its frequency content. The objective is to find the wave velocities $\nabla \varphi_{0}$, due to above-mentioned incident wave. The convolution integral from (2.15) can be used for this purpose as follows [33]:

$$
\nabla \varphi_{0}=\int_{-\infty}^{\infty} \boldsymbol{K}(\mathbf{r}, t-\tau) e^{-2 \pi^{2} s^{2} \tau^{2}} d \tau
$$

which is obtained by replacing $\tilde{\zeta}_{0}(\tau)$ with the Gaussian wave amplitude. The convolution in the timedomain can be calculated using multiplication in the frequency domain followed by Fourier transformation back into the time domain. This fact is employed to express the above convolution integral by:

$$
\mathcal{F}\left\{\nabla \varphi_{0}(\mathbf{r}, t)\right\}=\mathcal{F}\{\boldsymbol{K}(\mathbf{r}, t)\} \mathcal{F}\left\{e^{-2 \pi^{2} s^{2} t^{2}}\right\} .
$$

According to the definition of the Fourier-transform pair from (2.21), the Gaussian amplitude is transformed to the frequency domain as:

$$
\begin{aligned}
\mathcal{F}\left\{\zeta_{0}(t)\right\} & =\frac{1}{b} e^{-\frac{\omega_{e}^{2}}{a}}, \\
a & =8 \pi^{2} s^{2}, \\
b & =s \sqrt{2 \pi} .
\end{aligned}
$$

Since the impulse response function $K(\mathbf{r}, t)$ is known form $(2.20),(2.26)$ can be expressed as follows:

$$
\mathcal{F}\left\{\nabla \varphi_{0}(\mathbf{r}, t)\right\}=\frac{1}{2 \pi b} \boldsymbol{\Psi} e^{-\mathrm{i} k \alpha} e^{-\frac{\omega_{e}^{2}}{a}} .
$$

By taking the inverse Fourier transform, the body boundary condition in time domain is obtained:

$$
\nabla \varphi_{0}(\mathbf{r}, t)=\frac{1}{2 \pi b} \int_{-\infty}^{\infty} \Psi e^{-\mathrm{i} k \alpha} e^{-\frac{\omega_{e}^{2}}{a}} e^{\mathrm{i} \omega_{e} t} d \omega_{e} .
$$

In infinite water depth, a closed-form expression for this integral in terms of elementary functions is also given by [33], but in finite water depth the integral must be computed numerically. Details on how people have done this in the past are scarce in the literature, but indications are that it is typically done using Romberg integration to a specified tolerance. Here we instead apply the discrete Fast Fourier Transform (FFT). This can be carried out by selecting a suitable maximum frequency (Nyquist frequency), above which the integrand from (2.28) is zero to a desired tolerance, see Figure 2. A uniformly spaced set of discrete frequencies is then chosen between zero and Nyquist, and the discrete inverse FFT applied to compute the time-domain values. An example of the convergence of 
this method for computing the $x$ component of the wave velocity at a point in space, for the deepwater situation, is shown in Figure 3. Here the incidence angle is $\beta=\pi$ and the Froude number is $F r=0.4$. The analytical solution in this figure is from [33], and $d$ is the characteristic length of the geometry. Surprisingly, the method shows a second-order rate of convergence with increasing resolution $N_{f}$ instead of the exponential convergence which might be expected for what is essentially a spectral method. The reason for this is under investigation, but for practical calculations using the method, choosing $N_{f} \geq 10^{4}$ should ensure an accuracy of at least single-digit precision $\left(10^{-6}\right)$.

\subsection{Exploiting geometric symmetry}

As mentioned in the introduction, it is computationally convenient to exploit the geometric port/starboard ( $y=0$ plane) symmetry of the ship (if it exists), and perform the calculations on only half of the physical domain. In general, this requires splitting the body boundary condition in to symmetric and anti-symmetric components. If $\alpha$ in the velocity potential of the incident wave from (2.11) is considered, then:

$$
\begin{gathered}
e^{-\mathrm{i} k \alpha}=e^{-\mathrm{i} k(x \cos \beta+y \sin \beta)}=[\cos (k x \cos \beta)-\mathrm{i} \sin (k y \sin \beta)][\cos (k y \sin \beta)-\mathrm{i} \sin (k y \sin \beta)] \\
=\cos (k y \sin \beta)[\cos (k x \cos \beta)-\mathrm{i} \sin (k y \sin \beta)] \\
-\mathrm{i} \sin (k y \sin \beta)[\cos (k x \cos \beta)-\mathrm{i} \sin (k y \sin \beta)] .
\end{gathered}
$$

This decomposition can be used in (2.20) or (2.28) to obtain the symmetric and anti-symmetric boundary conditions as follows:

$$
-\boldsymbol{n} \cdot \nabla\left(\phi_{s}^{s}+\phi_{s}^{a}\right)=\left[n_{x} \frac{\partial}{\partial x}\left(\phi_{0}^{s}+\phi_{0}^{a}\right)+n_{y} \frac{\partial}{\partial y}\left(\phi_{0}^{s}+\phi_{0}^{a}\right)+n_{z} \frac{\partial}{\partial z}\left(\phi_{0}^{s}+\phi_{0}^{a}\right)\right] .
$$

where the superscripts $s$ and $a$ denote the symmetric and anti-symmetric potentials. Now, also considering the symmetry of the normal vector to the body boundary, the correct splitting of the boundary condition is:

$$
\begin{aligned}
-\boldsymbol{n} \cdot \nabla \phi_{s}^{s} & =n_{x} \frac{\partial \phi_{0}^{s}}{\partial x}+n_{y} \frac{\partial \phi_{0}^{a}}{\partial y}+n_{z} \frac{\partial \phi_{0}^{s}}{\partial z} \\
-\boldsymbol{n} \cdot \nabla \phi_{s}^{a} & =n_{x} \frac{\partial \phi_{0}^{a}}{\partial x}+n_{y} \frac{\partial \phi_{0}^{s}}{\partial y}+n_{z} \frac{\partial \phi_{0}^{a}}{\partial z} .
\end{aligned}
$$

For an arbitrary incident wave heading (except $\beta=0$ and $\beta=\pi$ ), two hydrodynamic problems should be solved with the corresponding body boundary conditions given by (2.30) and (2.31). Then the scattering wave forces in the surge, heave and pitch direction (the symmetric modes) are obtained only from the symmetric scattering potential $\phi_{s}^{s}$. In the same manner, the scattering wave forces in the roll, pitch and yaw directions (the anti-symmetric modes) are obtained only from the anti-symmetric scattering potential $\phi_{s}^{a}$.

\subsection{Following seas}

In the case where the waves are incident from abaft the beam (following seas), there are three different waves which produce the same encounter frequency with respect to the moving ship. These three incident waves can be explained qualitatively (with $\beta=0$ ) as follows:

1. A long wave which has a group velocity larger than $U$, and overtakes the vessel.

2. An intermediate wave with a phase velocity larger than $U$, but a group velocity less than $U$. This wave still appears to overtake the vessel, but due to the shorter wave length it produces the same encounter frequency. 

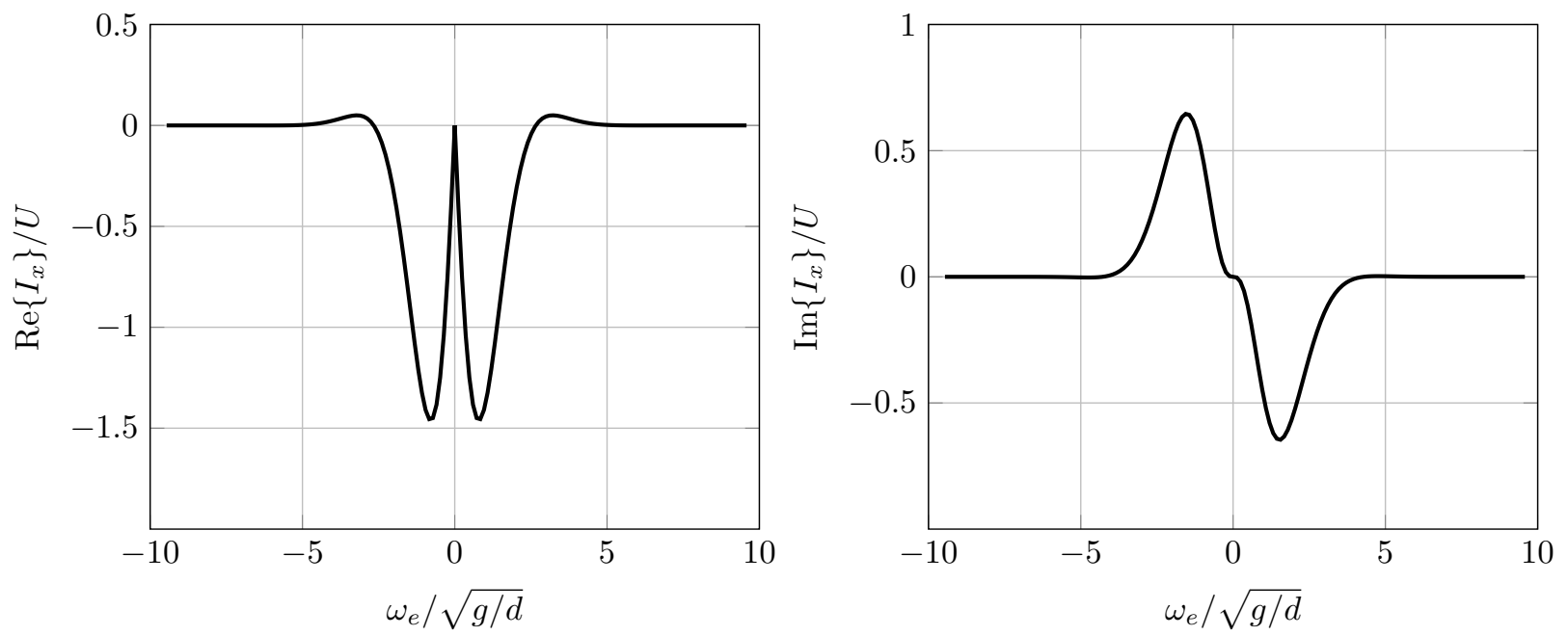

Figure 2: The real and imaginary parts of the integrand for the $x$ component of the incident wave velocity from (2.28). The Nyquist frequency $\omega_{e} / \sqrt{g / d}=9.46$ is chosen such that the integrand is less than $10^{-8}$ outside of this frequency range. The calculation is for the deep water condition with $F r=0.4$ and $\beta=\pi$.
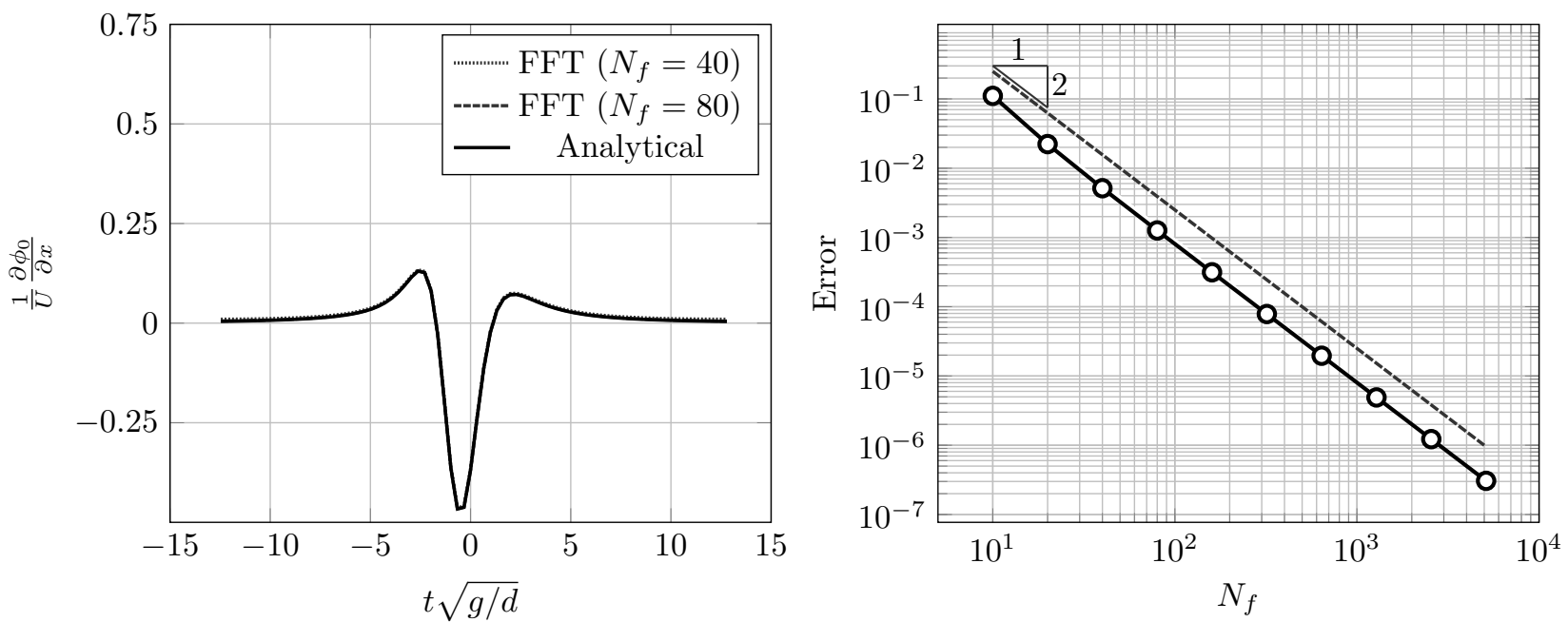

Figure 3: Convergence of the $x$ component of the incident wave velocity $\frac{\partial \phi_{0}}{\partial x}$ to the analytical solution given by [33]. The Fourier integral in (2.28) is approximated numerically using the FFT, with $N_{f}$ number of points in the frequency space. The calculation is for the deep water condition with $\mathrm{Fr}=0.4$ and $\beta=\pi$. 


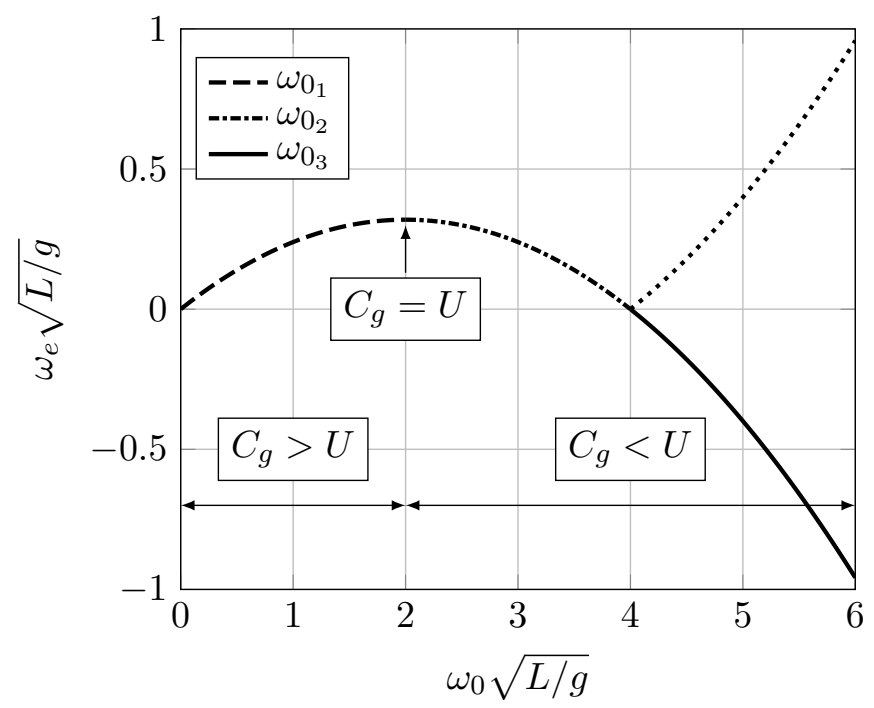

Figure 4: The relation between $\omega_{0}$ and $\omega_{e}$ for the following-seas condition $\beta=0$.

3. A wave with a phase velocity less than $U$ which therefore appears to come from ahead of the vessel, but again induces the same encounter frequency. Since it appears to come from ahead in this case, the velocity potential of the incident wave can be written:

$$
\phi_{0}(\mathbf{r}, t)=\operatorname{Re}\left\{\frac{\mathrm{i} A g}{\omega_{0}} \frac{\cosh k(z+h)}{\cosh k h} e^{\mathrm{i} k \alpha} e^{\mathrm{i} \omega_{e} t}\right\},
$$

which is obtained from (2.11) with $e^{-\mathrm{i} k \alpha} \rightarrow e^{\mathrm{i} k \alpha}$.

In general, it is in fact the induced ship velocity in the direction of wave propagation which should be compared to the phase velocity of the incident wave.

In order to describe how the body boundary condition for the following-seas situation should be calculated, consider again the relationship between the wave frequency $\omega_{0}$ and the encounter frequency $\omega_{e}$ :

$$
\omega_{e}=\omega_{0}-k U \cos \beta .
$$

Then using the dispersion relation $\omega_{0}^{2}=g k \tanh k h$ it is possible to write:

$$
\omega_{0}=\frac{g \tanh k h}{2 U \cos \beta}\left(1 \pm \sqrt{1-\omega_{e} \frac{4 U \cos \beta}{g \tanh k h}}\right) .
$$

According to the above-mentioned three scenarios, three possible wave frequencies for a single $\omega_{e}$ can be expressed as:

$$
\begin{array}{ll}
\omega_{0_{1}}=\frac{g \tanh k h}{2 U \cos \beta}\left(1-\sqrt{1-\omega_{e} \frac{4 U \cos \beta}{g \tanh k h}}\right), & 0 \leq \omega_{e} \leq \frac{g \tanh k h}{4 U \cos \beta}, \\
\omega_{0_{2}}=\frac{g \tanh k h}{2 U \cos \beta}\left(1+\sqrt{1-\omega_{e} \frac{4 U \cos \beta}{g \tanh k h}}\right), & 0 \leq \omega_{e} \leq \frac{g \tanh k h}{4 U \cos \beta}, \\
\omega_{0_{3}}=\frac{g \tanh k h}{2 U \cos \beta}\left(1+\sqrt{1+\left|\omega_{e}\right| \frac{4 U \cos \beta}{g \tanh k h}}\right), & 0 \leq\left|\omega_{e}\right| \leq \infty .
\end{array}
$$

In the deep-water condition where $\tanh k h=1$, these relations are plotted in Figure 4 . Note that $L$ in the figure is a characteristic length of the moving body, and $C_{g}$ is the wave group velocity. Here 
$\omega_{0_{1}}$ and $\omega_{0_{2}}$ correspond to the long and the intermediate waves respectively. Note that for $\omega_{0_{3}}$ the encounter frequency is negative due to the change in the phase of the incident wave. The absolute value of the corresponding encounter frequency is also shown in the figure by a dotted line. The wave frequency at which the maximum positive encounter frequency occurs is given by:

$$
\bar{\omega}_{0}=\frac{g \tanh k h}{2 U \cos \beta},
$$

The corresponding encounter frequency can also be calculated as:

$$
\bar{\omega}_{e}=\frac{g \tanh k h}{4 U \cos \beta} .
$$

Note that $\bar{\omega}_{e}$ is the maximum encounter frequency which occurs in regions $\omega_{01}$ and $\omega_{01}$. The encounter frequency can of course reach infinitely large values in the $\omega_{03}$ region.

For the arbitrary-depth condition, the dispersion relation can be used again to find this wave frequency iteratively. This reduces to finding the following wave number:

$$
k=\frac{g \tanh k h}{(2 U \cos \beta)^{2}},
$$

which can be used afterwards to obtain the range of the encounter frequency from (2.34)-(2.36).

As mentioned earlier, three time-domain diffraction problems should be solved in order to obtain the frequency-domain solutions in the $\omega_{01}, \omega_{02}$ and $\omega_{03}$ regions. The velocities of the incident waves for these problems can be calculated from the following convolution integrals:

$$
\nabla \varphi_{0 m}(\mathbf{r}, t)=\int_{-\infty}^{\infty} \boldsymbol{K}_{m}(\mathbf{r}, t-\tau) \tilde{\zeta}_{0_{m}}(\tau) d \tau, \quad m=1 \cdots 3
$$

in which:

$$
\begin{aligned}
& \tilde{\zeta}_{0_{1,2}}= \begin{cases}e^{\mathrm{i} \omega_{e} t} & 0 \leq \omega_{e} \leq \bar{\omega}_{e} \\
0 & \omega_{e}>\bar{\omega}_{e}\end{cases} \\
& \tilde{\zeta}_{0_{3}}=e^{-\mathrm{i} \omega_{e} t} \quad 0<\omega_{e}<\infty .
\end{aligned}
$$

And similar to (2.20) three impulse response functions can be obtained:

$$
\begin{aligned}
\boldsymbol{K}_{1,2}(\mathbf{r}, t) & =\frac{1}{2 \pi} \int_{-\infty}^{\bar{\omega}_{e}} \boldsymbol{\Psi}_{1,2} e^{-\mathrm{i} k \alpha} e^{\mathrm{i} \omega_{e} t} d \omega_{e}, \\
\boldsymbol{K}_{3}(\mathbf{r}, t) & =\frac{1}{2 \pi} \int_{-\infty}^{\infty} \boldsymbol{\Psi}_{3} e^{\mathrm{i} k \alpha} e^{\mathrm{i} \omega_{e} t} d \omega_{e}
\end{aligned}
$$

in which $\boldsymbol{\Psi}_{1,2}$ and $\boldsymbol{\Psi}_{3}$ are calculated from (2.18) with the respective values of $\omega_{0_{1}}, \omega_{0_{2}}$ and $\omega_{0_{3}}$. For the case of a pseudo-impulsive incident wave elevation, the wave velocities for the body boundary condition can be calculated from:

$$
\begin{aligned}
\nabla \varphi_{0_{1,2}}(\mathbf{r}, t) & =\frac{1}{2 \pi b} \int_{-\infty}^{\bar{\omega}_{e}} \boldsymbol{\Psi}_{1,2} e^{-\mathrm{i} k \alpha} e^{-\frac{\omega_{e}^{2}}{a}} e^{\mathrm{i} \omega_{e} t} d \omega_{e} \\
\nabla \varphi_{0_{3}}(\mathbf{r}, t) & =\frac{1}{2 \pi b} \int_{-\infty}^{\infty} \boldsymbol{\Psi}_{3} e^{\mathrm{i} k \alpha} e^{-\frac{\omega_{e}^{2}}{a}} e^{\mathrm{i} \omega_{e} t} d \omega_{e}
\end{aligned}
$$

As before, all three integrals are calculated numerically using the discrete inverse Fourier transform. Note that for the case $m=1$ and $m=2$, the numerical transform to calculate the wave velocity, will 
be performed assuming that the highest frequency is equal to $\bar{\omega}_{e}$. The frequency-domain data above this frequency limit is just zero. Later after the Fourier transformation of the time-domain results for these two cases, the frequency-domain solutions at higher frequencies than $\bar{\omega}_{e}$ will be discarded.

For each specific heading in the following-seas condition, three distinct scattering velocity potentials should be calculated, to obtain the results in the corresponding frequency ranges $\omega_{01}, \omega_{02}$ and $\omega_{03}$. This is achieved by solution to three initial boundary value problems subject to the above three body boundary conditions respectively. If symmetry is exploited in the following-sea cases, for each arbitrary heading (except $\beta=0$ ), 6 hydrodynamic scattering problems should be solved in order to obtain the wave excitation forces. This is in contrast to the 2 diffraction problems for each arbitrary heading in the case of wave incident from ahead of the beam.

\subsection{Wave excitation forces}

The total diffraction flow field around the body $\phi_{d}$, is comprised of the flow due to the incident wave $\phi_{0}$ and the scattered waves $\phi_{s}$. This can simply be written as $\phi_{d}=\phi_{0}+\phi_{s}$. The part of the excitation force due to $\phi_{s}$ can be calculated by integration of the linear pressures on the surface of the body. This linear dynamic wave pressure is obtained from the Bernoulli equation keeping only the first-order terms:

$$
p(x, y, z, t)=-\rho\left\{\left(\frac{\partial}{\partial t}-\boldsymbol{W} \cdot \nabla\right) \phi_{d}+\nabla \phi_{b}^{\prime} \cdot \nabla \phi_{d}\right\} .
$$

The resulting time-domain forces then can be Fourier transformed to get the corresponding forces in the frequency domain. The relationship between the wave excitation forces due to $\phi_{s}$ in the time- and frequency-domains is reviewed here. In general for a linear system, the wave force in direction $j$ due to a monochromatic incident wave with the encounter frequency $\omega_{e}$ can be expressed by:

$$
F_{j}(t)=A_{f} \cos \left(\omega_{e} t+\theta_{0}\right)=\operatorname{Re}\left\{A_{f} e^{\mathrm{i} \theta_{0}} e^{\mathrm{i} \omega_{e} t}\right\}=\operatorname{Re}\left\{X_{j} e^{\mathrm{i} \omega_{e} t}\right\} .
$$

In which $A_{f}$ is the amplitude of the wave force in time domain, and $\theta_{0}$ is the phase angle. A diffraction impulse response function $K_{j D}(t-\tau)$, can be defined for the wave forces in direction $j$, based on the impulsive incident wave elevation. Then the wave force in direction $j$ due to any arbitrary incident wave amplitude $\tilde{\zeta}_{0}$ can be calculated by convolution:

$$
\tilde{F}_{j}(t)=\int_{-\infty}^{\infty} K_{j D}(t-\tau) \tilde{\zeta}_{0}(\tau) d \tau
$$

If the wave elevation from (2.14) is inserted, and also by the change of variable $t-\tau=t^{\prime}$, then:

$$
F_{j}(t)=\operatorname{Re}\left\{X_{j} e^{\mathrm{i} \omega_{e} t}\right\}=\operatorname{Re}\left\{\int_{-\infty}^{\infty} K_{j D}(t-\tau) e^{\mathrm{i} \omega_{e} \tau} d \tau\right\}=\operatorname{Re}\left\{e^{\mathrm{i} \omega_{e} t} \int_{-\infty}^{\infty} K_{j D}(\tau) e^{\mathrm{i} \omega_{e} \tau} d \tau\right\} .
$$

The phasors of two equal sinusoids should be the same, which leads to the well-known relation:

$$
X_{j}=\int_{-\infty}^{\infty} K_{j D}(\tau) e^{\mathrm{i} \omega_{e} \tau} d \tau=\mathcal{F}\left\{K_{j D}(t)\right\} .
$$

If the convolution integral is implemented instead using multiplication in the frequency domain then (2.47) can be written equivalently as follows:

$$
F_{j}(t)=\mathcal{F}^{-1}\left\{\mathcal{F}\left\{K_{j D}(t)\right\} \mathcal{F}\left\{\tilde{\zeta}_{0}(t)\right\}\right\} .
$$

By taking the Fourier transform the following equation is reached:

$$
X_{j}=\frac{\mathcal{F}\left\{F_{j}(t)\right\}}{\mathcal{F}\left\{\tilde{\zeta}_{0}(t)\right\}} .
$$



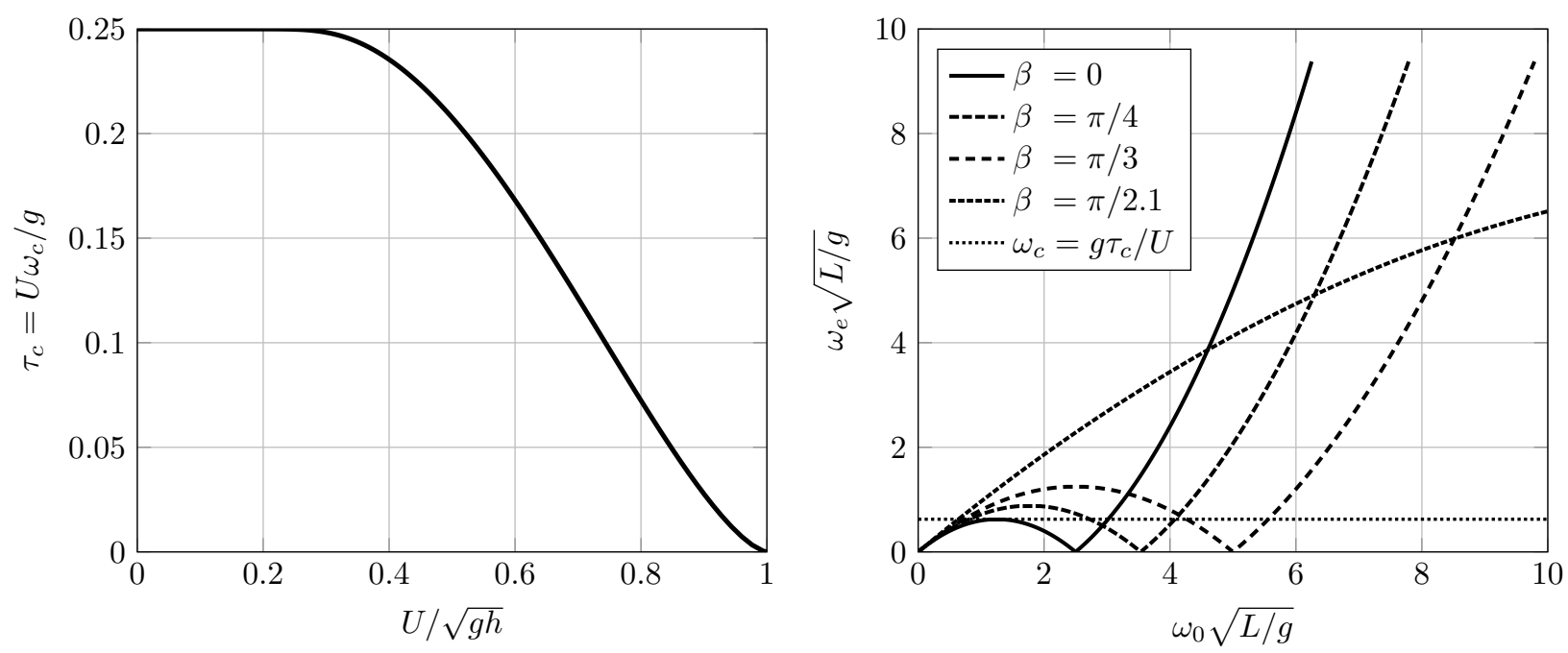

Figure 5: The non-dimensional critical encounter frequency $\tau_{c}$ as a function of the depth Froude number (left), and the relation between the critical frequency and the wave frequencies when $0 \leq \beta<$ $\pi / 2$ (right)

The time-domain wave force $F_{j}(t)$, obtained from the simulation and equation (2.45), can be transformed to the frequency domain using (2.50). Here the denominator will be the Fourier transform of the pseudo-impulsive wave elevation given by (2.24). Note that the Fourier-transforms are in terms of the encounter frequency $\omega_{e}$.

The contribution to the exciting forces due to the incident wave velocity potential $\phi_{0}$ (which is also called the Froude-Krylov force), can be computed directly in the frequency domain using the corresponding linear wave solution. For example for the first term containing the time derivative of the incident wave in $(2.45)$ :

$$
\begin{aligned}
& \bar{p}_{0}^{s}=\rho g \frac{\omega_{e}}{\omega_{0}} \frac{\cosh k(z+h)}{\cosh k h} e^{-\mathrm{i} k x \cos \beta} \cos (k y \sin \beta), \\
& \bar{p}_{0}^{a}=-\mathrm{i} \rho g \frac{\omega_{e}}{\omega_{0}} \frac{\cosh k(z+h)}{\cosh k h} e^{-\mathrm{i} k x \cos \beta} \sin (k y \sin \beta) .
\end{aligned}
$$

where $\bar{p}_{0}^{s}$ and $\bar{p}_{0}^{a}$ are the symmetric and anti-symmetric part of the incident wave pressure in the frequency domain. Other terms containing the gradient of the incident wave can be calculated in the same manner in the frequency domain. The resulting forces are added to the frequency-domain solutions from the scattering problem which have been calculated using (2.50). For the following-sea conditions, the Froude-Krylov forces have to be determined separately for each of the three regions $\omega_{01}, \omega_{02}$ and $\omega_{03}$.

\subsection{Treatment of the critical frequency}

At steady forward-speed, there is always a critical encounter frequency $\omega_{c}$ where the group velocity of a wave generated by the ship, $C_{g}$, is equal to the forward speed of the vessel. The influence of this frequency will thus be important for both the radiation and the diffraction problems. In arbitrary water depth this situation can be expressed as:

$$
C_{g}=\frac{1}{2} \frac{\omega_{0}}{k}\left(1+\frac{2 k h}{\sinh 2 k h}\right)=U,
$$

where $\omega_{0}$ and $k$ are the wave frequency and the wave number at the critical point, and can be calculated implicitly from this equation and the dispersion relation. The corresponding critical encounter 
frequency then is obtained from:

$$
\omega_{c}=\omega_{0}-k U .
$$

In non-dimensional form, this is expressed as a relation between $\tau_{c}=\omega_{c} U / g$ and the depth Froude number $F r=U / \sqrt{g h}$, which is plotted in Figure 5 (left). Note that $\tau_{c}$ is independent of the incident wave angle. When $\beta=0$, the critical frequency $\omega_{c}$ coincides with the boundary between the $\omega_{01}$ and $\omega_{02}$ regions, and there are two wave frequencies corresponding to $\omega_{c}$. For all other heading angles $0<\beta<\pi / 2$, the critical frequency appears at three wave frequencies, as also shown in Figure 5 (right).

At the critical frequency, where the group velocity of the ship-generated waves equals the ship speed, that portion of the scattered energy which is sent directly forward becomes partially trapped at the body, producing (theoretically) large forces. In a pseudo-impulsive time-domain problem, there will always be some energy in these waves, leading to a slowly-decaying tail in the excitation force signal. Extremely long simulations, and correspondingly large spatial domains, would be required to fully capture this signal numerically. Instead, we simulate for a time corresponding to

$$
\bar{t}_{m}=t_{m} \sqrt{g / L_{t}} \approx 15 .
$$

where $L_{t}$ is length-scale of the computational domain. Then, following [16] and as discussed in more detail by [26], we make a least-squares fit of the signal to the function

$$
F_{a}(t)=\frac{1}{t}\left[a_{1} \sin \left(\omega_{c} t\right)+a_{2} \cos \left(\omega_{c} t\right)\right] .
$$

The Fourier transform of (2.56) is given by:

$$
\int_{t_{m}}^{t_{\infty}} \frac{1}{t}\left[a_{1} \sin \left(\omega_{c} t\right)+a_{2} \cos \left(\omega_{c} t\right)\right] e^{-i \omega_{e} t} d t,
$$

which is combined with the numerical Fourier transform (FFT) of the computed signal to give the coefficients in the frequency-domain. In the Results section some plots are also presented to show this asymptotic behavior at the critical frequency.

\section{$3 \quad$ Numerical method}

The initial boundary-value problem posed in Section 2, is solved here using fourth-order finitedifference approximations. The computational domain is fully discretized in three dimensions. Bodyfitted and curvilinear overlapping grids are built to define the domain around the body. The finitedifference computation is carried out in the rectangular mapped domain which is obtained by a numerical or analytical transformation (in the case of close-form geometries). Two layers of ghost points are used to be able to use the fourth-order and centered finite-difference schemes also at the boundaries. Interpolation points are defined in overlapping regions to communicate the solution between the component grids.

At the free surface $S_{f}$, the Dirichlet condition is applied to specify the value of the velocity potential $\phi_{s}$ at $z=0$. A non-homogeneous Neumann condition is employed at $S_{b}$ to apply the body boundary condition (2.10). At $S_{d}$ and $S_{\infty}$, homogeneous Neumann conditions are applied. First the Laplace equation (2.1) subjected to above-mentioned boundary conditions is solved to obtain the velocity potential $\phi_{s}$ everywhere in the domain including the free surface, and the body surface. This is performed by finding the solution to the following linear system of equations:

$$
[\boldsymbol{A}] \phi_{s}=[b] .
$$

In which $[\boldsymbol{A}]$ is the matrix containing the coefficients for the Laplace equation, and the applied boundary conditions. The relevant forcing due to the corresponding boundary conditions and the Laplace 
equation is also placed in the vector $[b]$. The solution at the free surface is then updated by time integration of the free-surface equations (2.7) and (2.8), using the explicit fourth-order Runge-Kutta method. To ensure numerical stability, the convective derivatives in the free-surface conditions are calculated using an upwind biased scheme. Numerical stability also requires special treatment of the ghost points at the boundaries of the free surface. More details about the developed numerical methodology can be found in [26], and [36].

\section{Results}

For the validation of the numerical method, three geometries are considered. First the wave excitation forces are calculated for a submerged 2D circular cylinder which moves with a constant forward speed. Both head seas and following seas are considered. Next a submerged and moving sphere will be subjected to the incident wave with a couple of different heading angles which are all ahead of the beam. By this geometry, the ability of the proposed method to include the symmetry of the body for any arbitrary heading, is also verified. Finally an overlapping grid is built for a bulk carrier, and the wave excitation forces and the ship motions are calculated in both head and following seas.

\subsection{Submerged circular cylinder}

The circular cylinder is submerged with a submergence depth of $\tilde{h}=2 a$, where $a$ denotes the radius of the cylinder. The body has a Froude number given by $F r=U / \sqrt{g a}=0.4$, and the water depth is $h=10 a$. A close-up of the overlapping grid for this geometry is also shown in Figure 6 . Note that the length of the computational grid is $L_{t}=140 a$. Two incident wave angles are considered, $\beta=\pi$ and $\beta=0$. In Figure 7, the time-domain pseudo-impulsive incident wave together with the scattering forces in the surge and heave directions are presented. In the figure, $D$ denotes the diameter of the cylinder. Note also that when $\beta=0$, for each force direction, three time simulations are shown in the figure.

The magnitude of the wave excitation forces are plotted in Figure 8, where a comparison is also made with the numerical solution provided by [37]. The solution method in [37] is in fact a combination of the finite element, and the boundary element methods. Note that the analytical results in [37] are obtained using the Neumann-Kelvin linearization $\phi_{b}^{\prime}=0$ for the free-surface conditions. Moreover the steady flow used in [37] is the linear solution to the wave resistance problem. For these simulations, instead the double-body flow $\left(\phi_{b}^{\prime}=\phi_{d b}\right)$ will be used as the base flow in the calculation of the wave excitation forces from (2.45). These results are non-dimensionalized by $\rho \pi a^{2} A \exp \left(-\nu_{0} \tilde{h}\right)$, where $\nu_{0}=\omega_{0}^{2} / g$, and $A$ is the amplitude of the incident wave. The critical frequency for the head-seas situations occurs at $\nu_{0}^{c} a=0.2674$, whereas for the following-seas case it occurs at $\nu_{0}^{c} a=1.5625$ and $\nu_{0}^{c}=9.1096$ respectively. Note also that the results from [37] are reproduced by digitization of the original plots. 


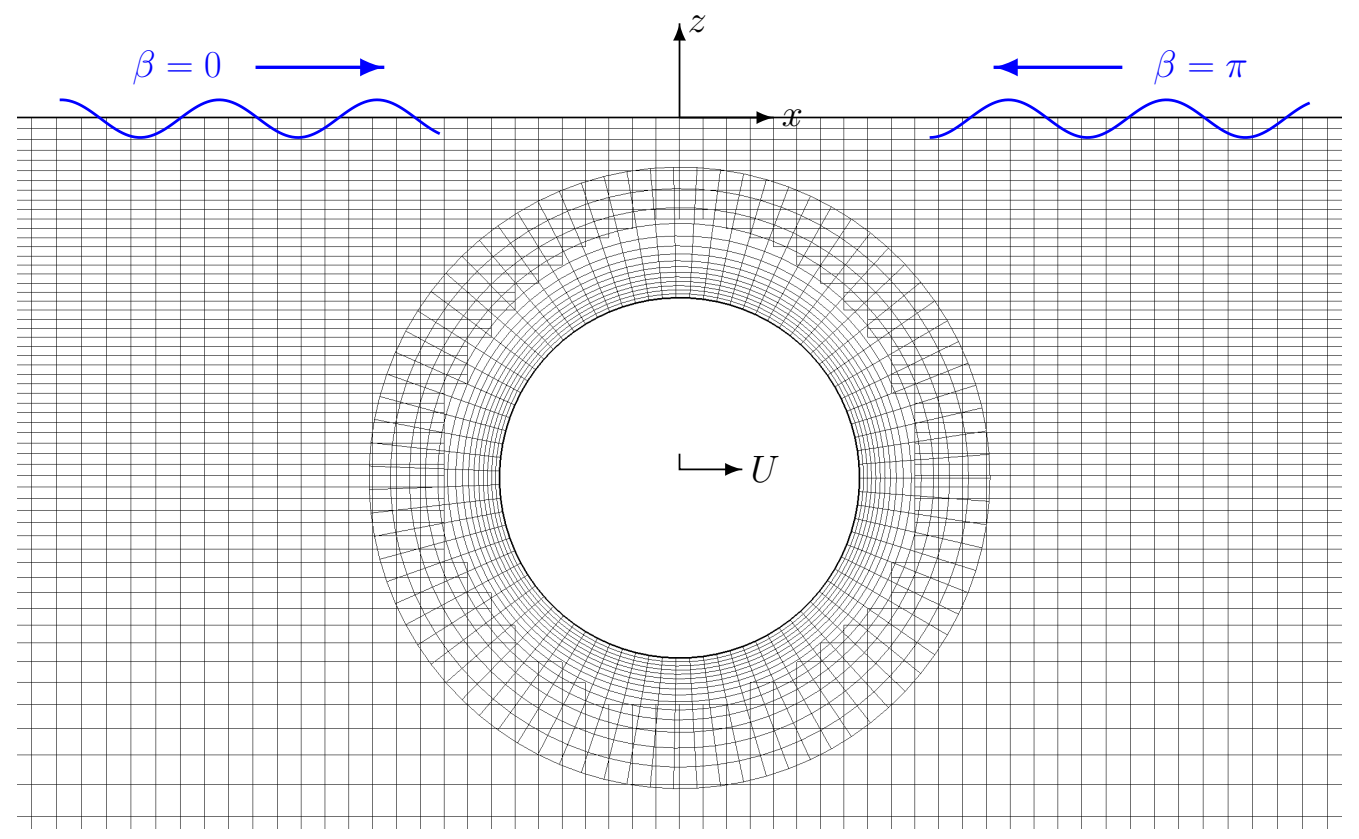

Figure 6: The overlapping grid for the 2D submerged circular cylinder 

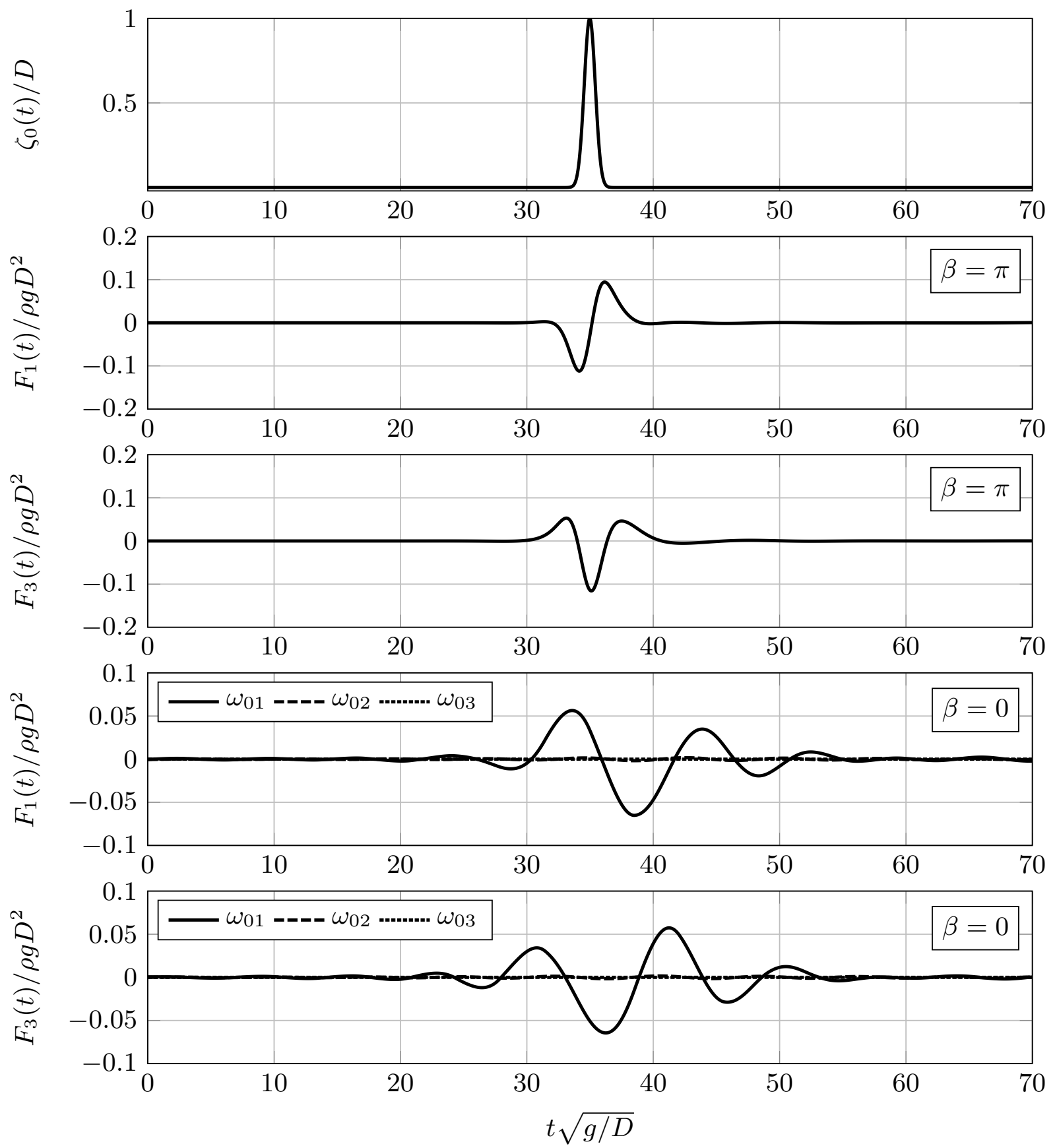

Figure 7: The pseudo-impulsive incident wave elevation $\zeta_{0}(t)$ given by $(2.24)$, and the time-domain scattering wave forces in the surge and heave directions for the submerged cylinder with $\mathrm{Fr}=0.40$. The forces are shown for both $\beta=\pi$ and $\beta=0$. Note that three time-domain simulations are shown for the following-seas case corresponding to the $\omega_{01}, \omega_{02}$ and $\omega_{03}$ regions in the frequency domain. 

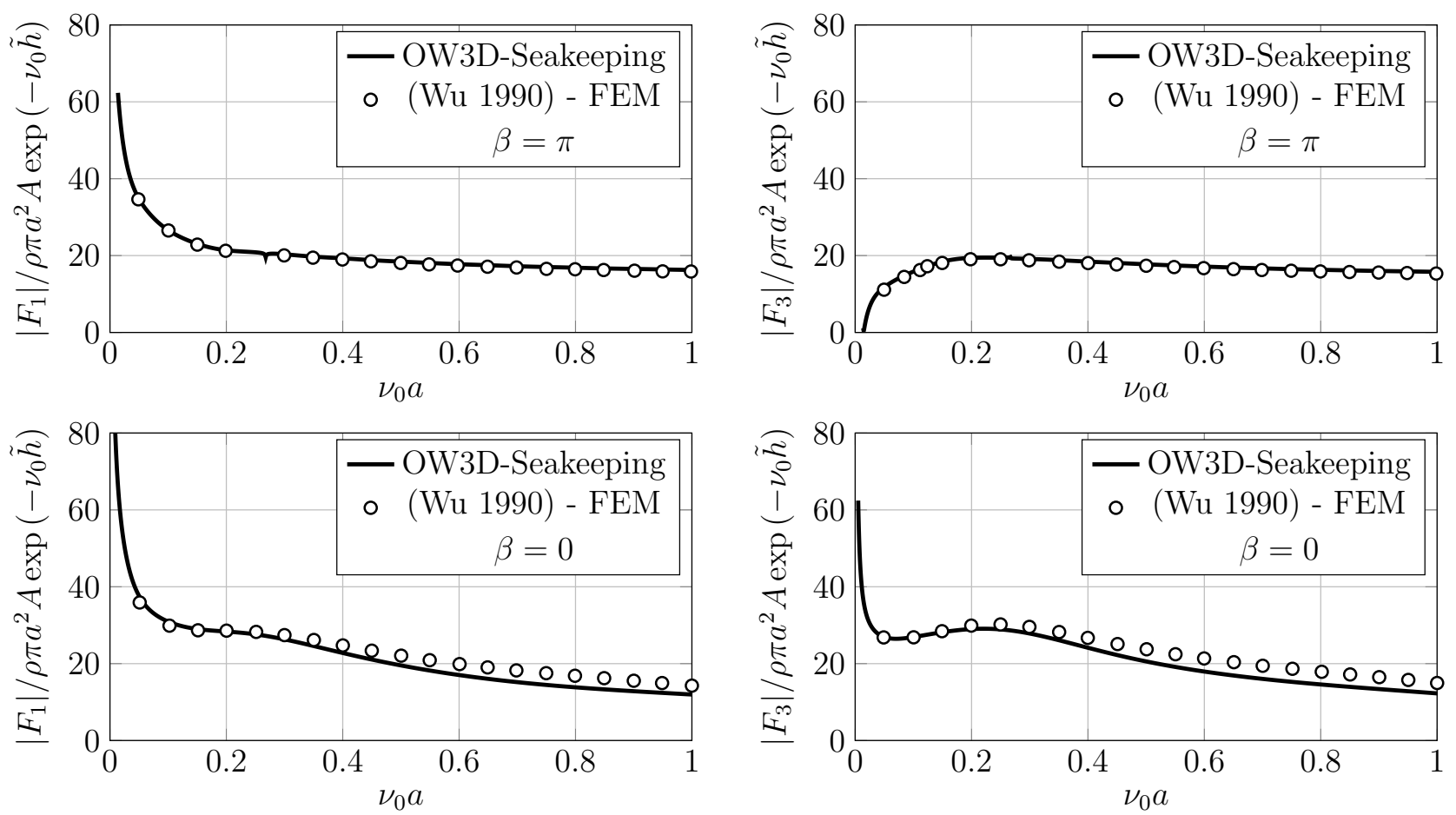

Figure 8: The magnitude of the wave excitation forces in surge $\left|F_{1}\right|$ and heave $\left|F_{3}\right|$, for the circular cylinder with $F r=0.4$. The results are both for the head-seas $(\beta=\pi)$ and the following-seas $(\beta=0)$ conditions. 


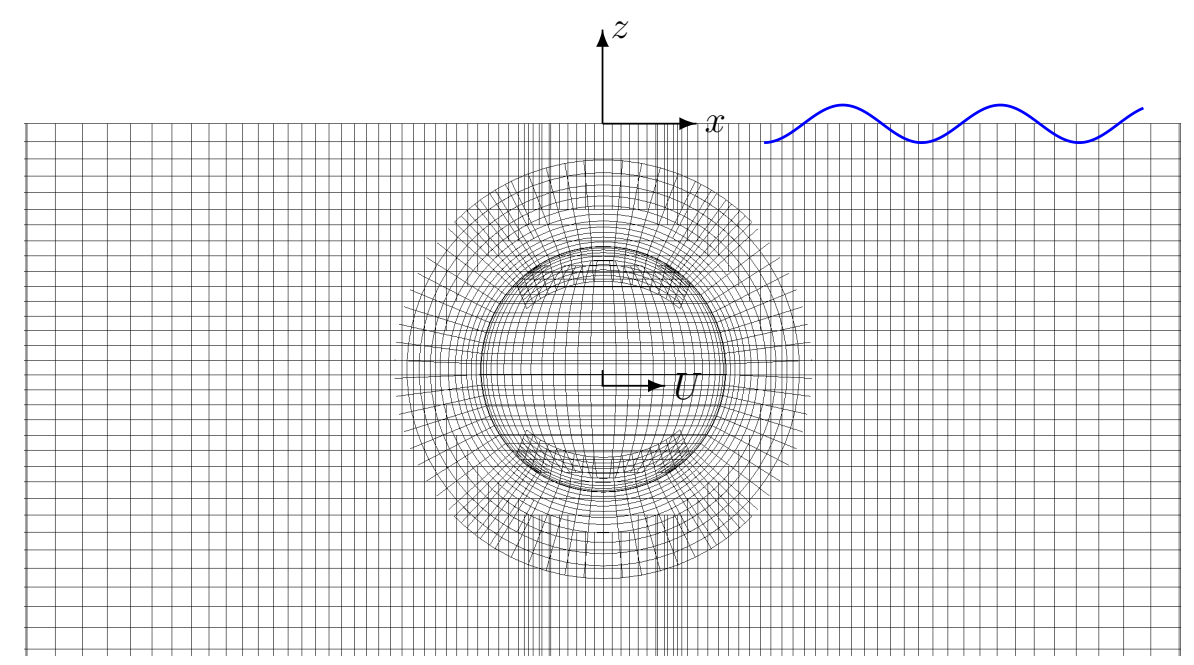

Figure 9: Part of the 3D overlapping grid for the submerged sphere (side view).

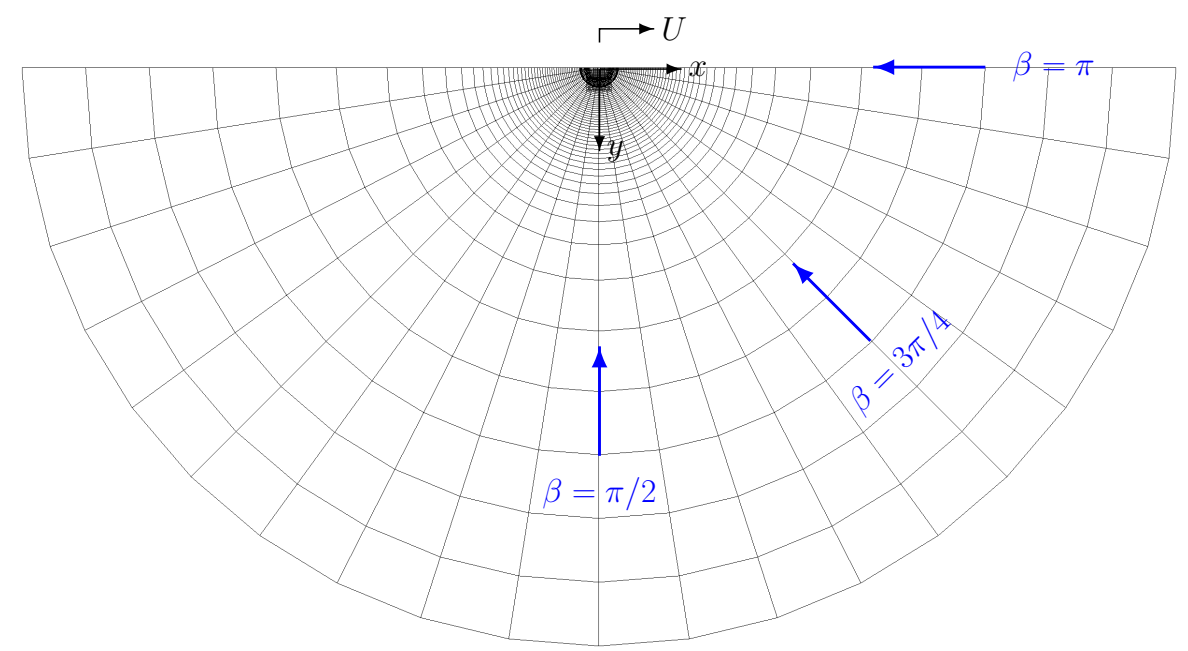

Figure 10: Part of the 3D overlapping grid for the submerged sphere (top view).

\subsection{Submerged sphere}

The sphere is submerged with the submergence depth of $\tilde{h}=2 a$, where $a$ is the radius. The body is moving with a speed given by the Froude number $F r=U / \sqrt{g a}=0.4$. Part of the overlapping grid built for this geometry is shown in Figure 9 and 10. The computational grid has a depth $h=14 a$ and the radius of the cylindrical background grid is $R_{c}=60 a$. The numerical calculations are compared with the analytical solution due to [38], which is for the deep-water condition. In addition, note that the steady flow used in [38] to calculate the excitation forces is in fact the solution to the linearized wave resistance problem. For the simulations in the developed solver, instead the doublebody flow $\left(\phi_{b}^{\prime}=\phi_{d b}\right)$ will be employed as the base flow in the calculation of the wave excitation forces from (2.45). Moreover it should be emphasized that the analytical solution provided by [38] is based on the Neumann-Kelvin linearization $\phi_{b}^{\prime}=0$ of the free-surface conditions $(2.7)-(2.8)$.

Three incident wave angels are considered: $\beta=\pi, 3 \pi / 4$ and $\pi / 2$. In Figure 11, the pseudoimpulsive incident wave elevation $\zeta_{0}(t)$ together with the scattering wave forces in the time domain in the surge, sway and heave directions are presented for the case of $\beta=\pi / 2$ (as an example). The asymptotic extrapolation of the tail of the force signal which oscillates at the critical frequency is also plotted by the dash-dotted lines. The critical frequency for $\beta=\pi, 3 \pi / 4$ and $\pi / 2$ occurs at $\nu_{0} a=0.2680, \nu_{0} a=0.2937$ and $\nu_{0} a=0.3906$ respectively. In the figure $D$ is the diameter of the 

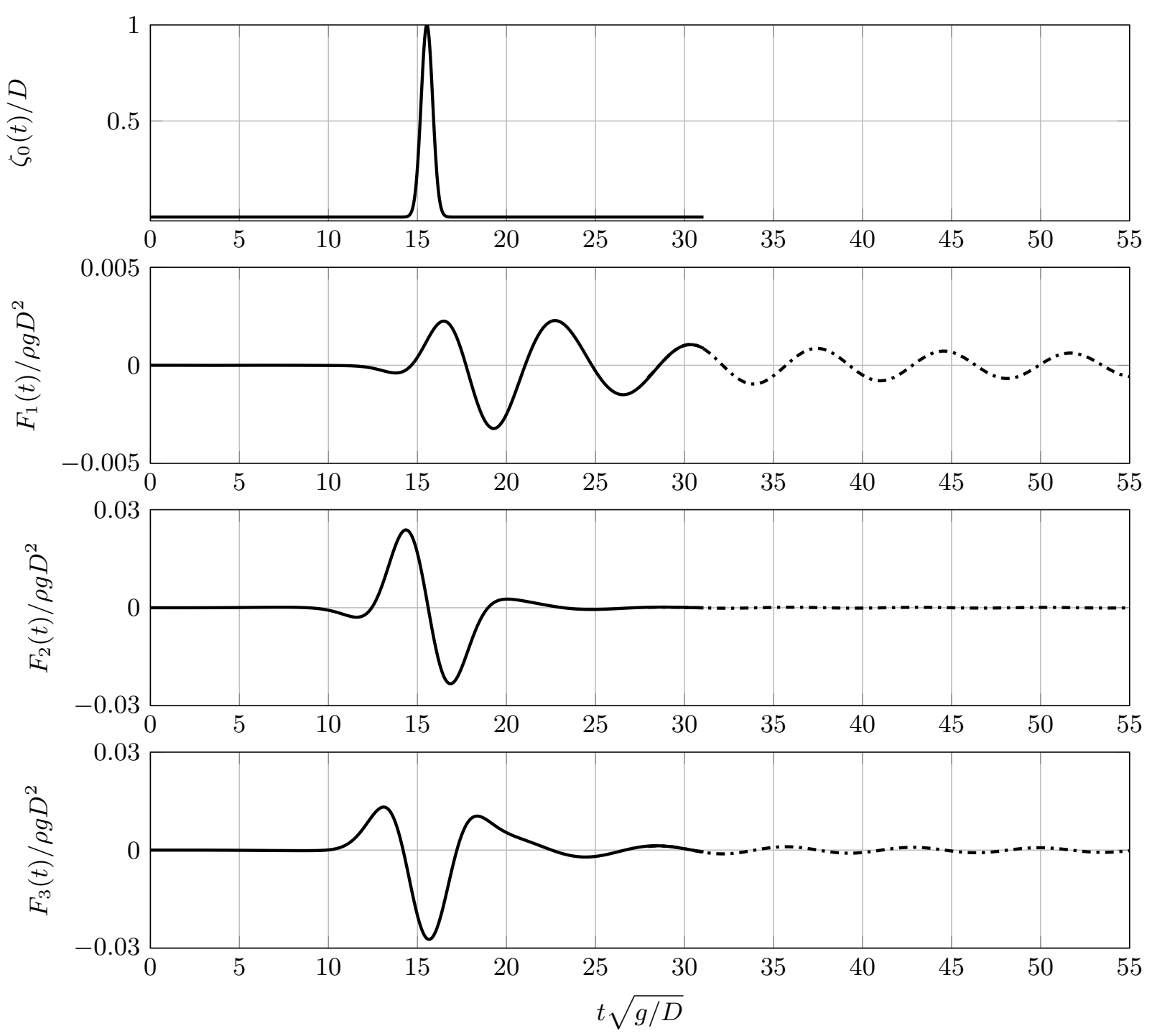

Figure 11: The pseudo-impulsive incident wave elevation $\zeta_{0}(t)$ given by $(2.24)$, and the time-domain scattering wave forces in the surge, sway and heave directions for the submerged sphere, $\beta=0.5 \pi$ and $F r=0.40$. The asymptotic extrapolation at the critical frequency is also shown for each force direction by the dash-dotted line.

sphere. As can also be seen from Figure 10, the simulation is performed using half of the geometry. The scattering forces are obtained by integrating the pressure terms from (2.45) over the surface of the sphere. The frequency-domain results including the real and the imaginary parts of the excitation forces, are shown in Figures 12 to 19, for all three incident wave angles. Note that the frequencydomain results are non-dimensionalized by $\rho g \pi a^{3} \nu A$, where $\nu=\omega_{e}^{2} / g$. 

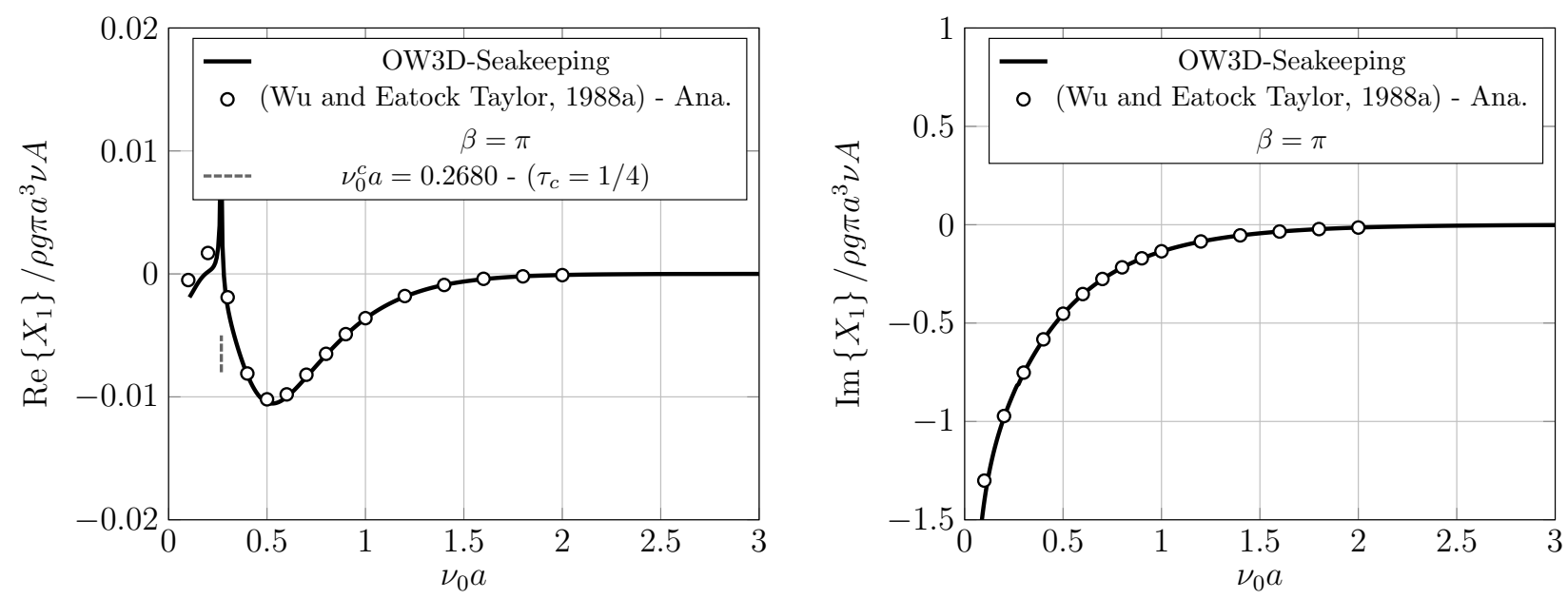

Figure 12: The wave excitation force in surge, $X_{1}$, on the submerged sphere $\beta=\pi, F r=0.40$
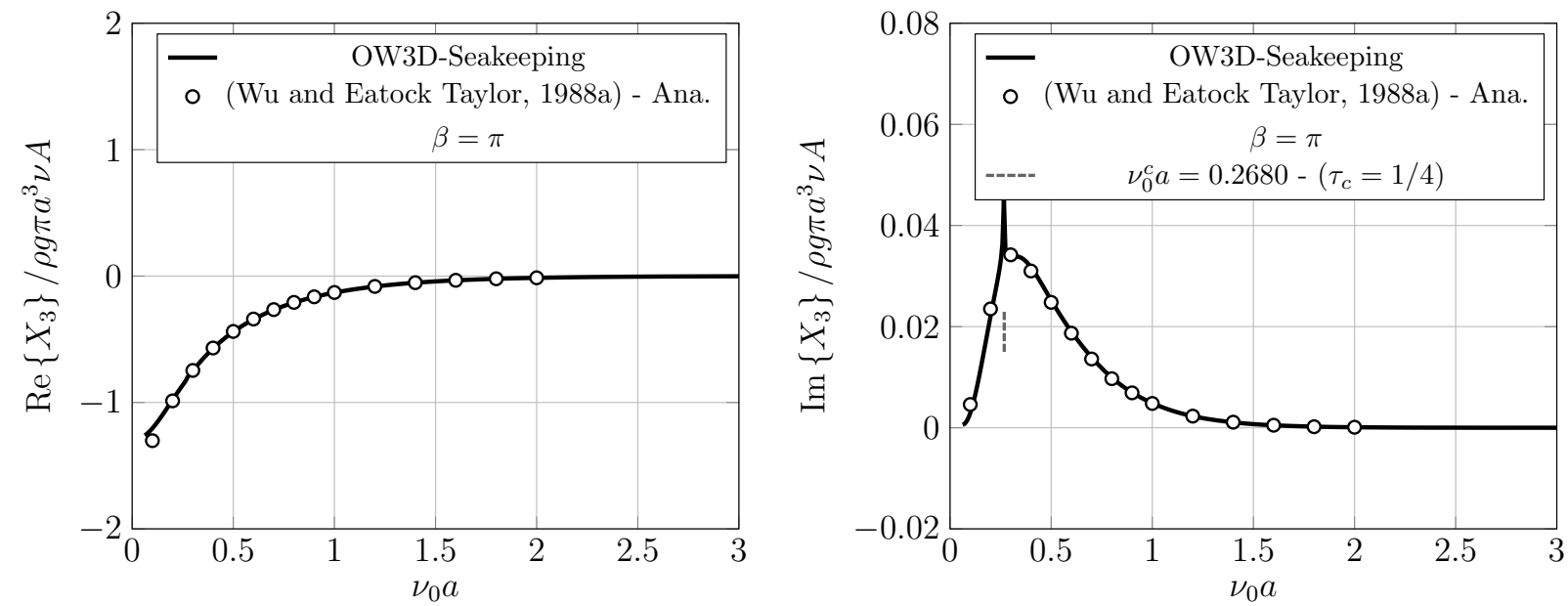

Figure 13: The wave excitation force in heave, $X_{3}$, on the submerged sphere $\beta=\pi, F r=0.40$ 

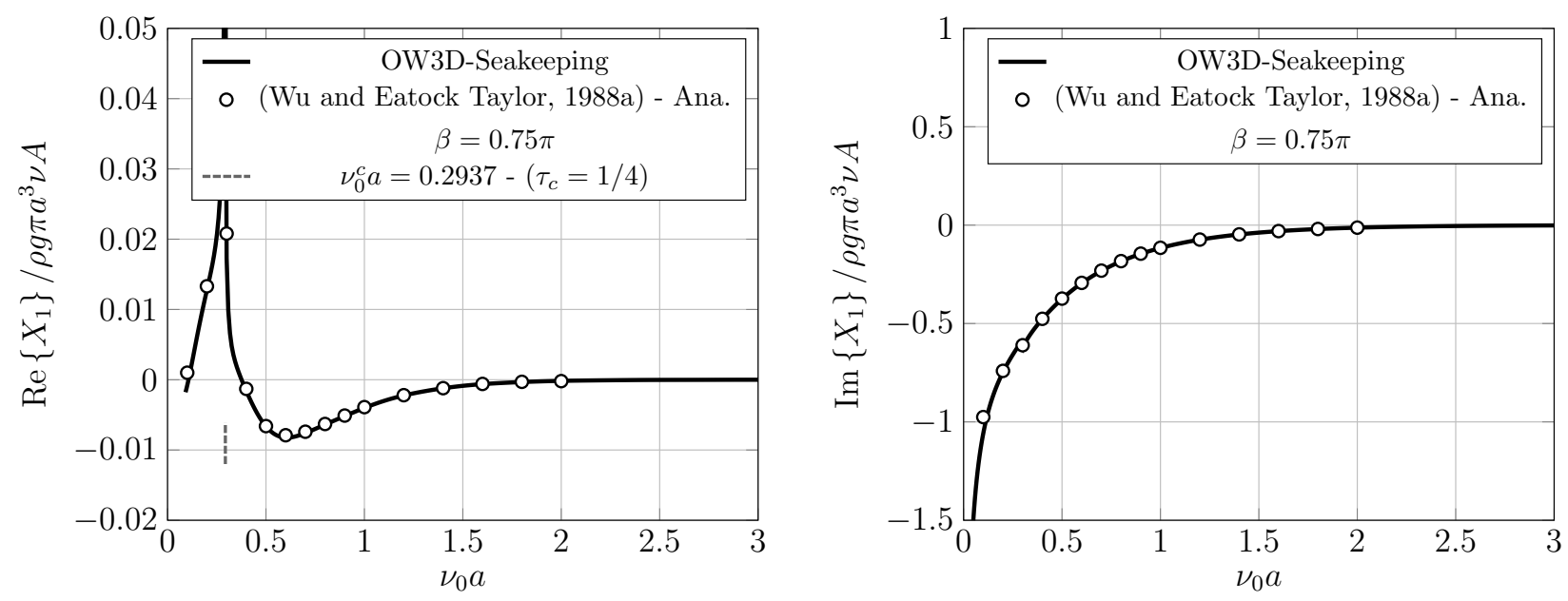

Figure 14: The wave excitation force in surge, $X_{1}$, on the submerged sphere $\beta=3 \pi / 4, F r=0.40$
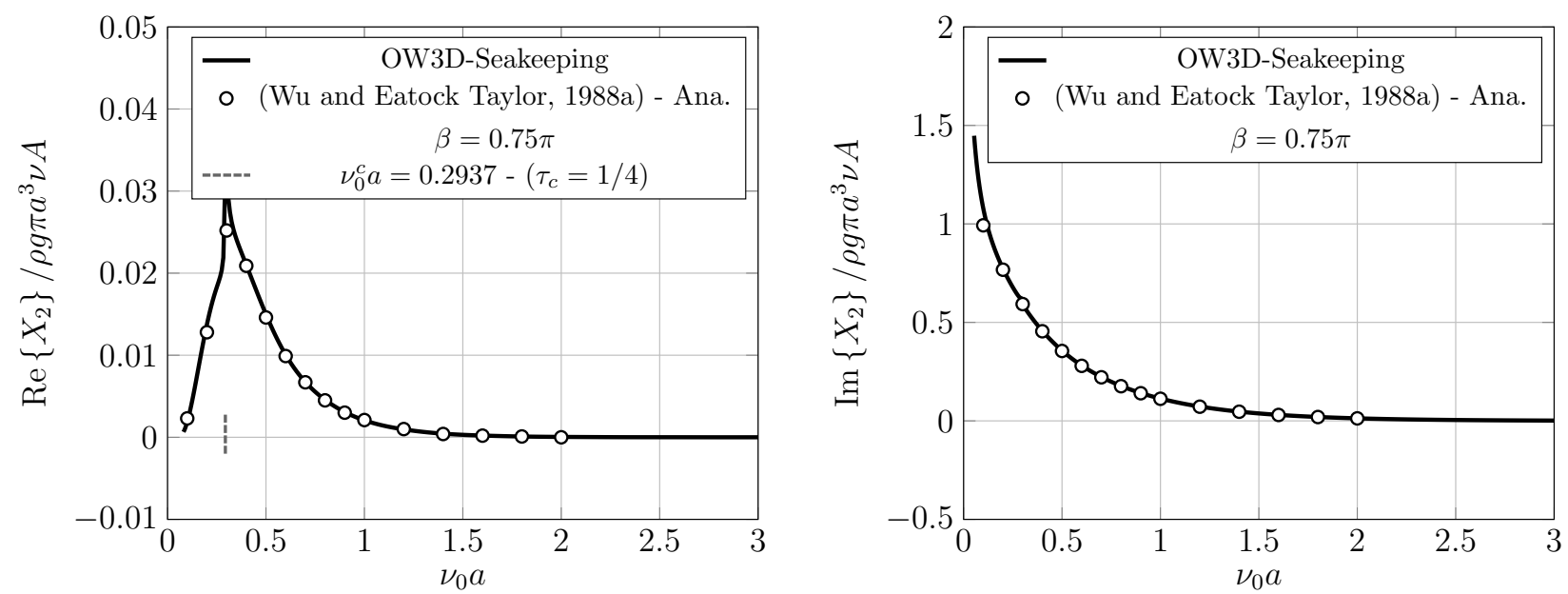

Figure 15: The wave excitation force in sway , $X_{2}$, on the submerged sphere $\beta=3 \pi / 4, F r=0.40$
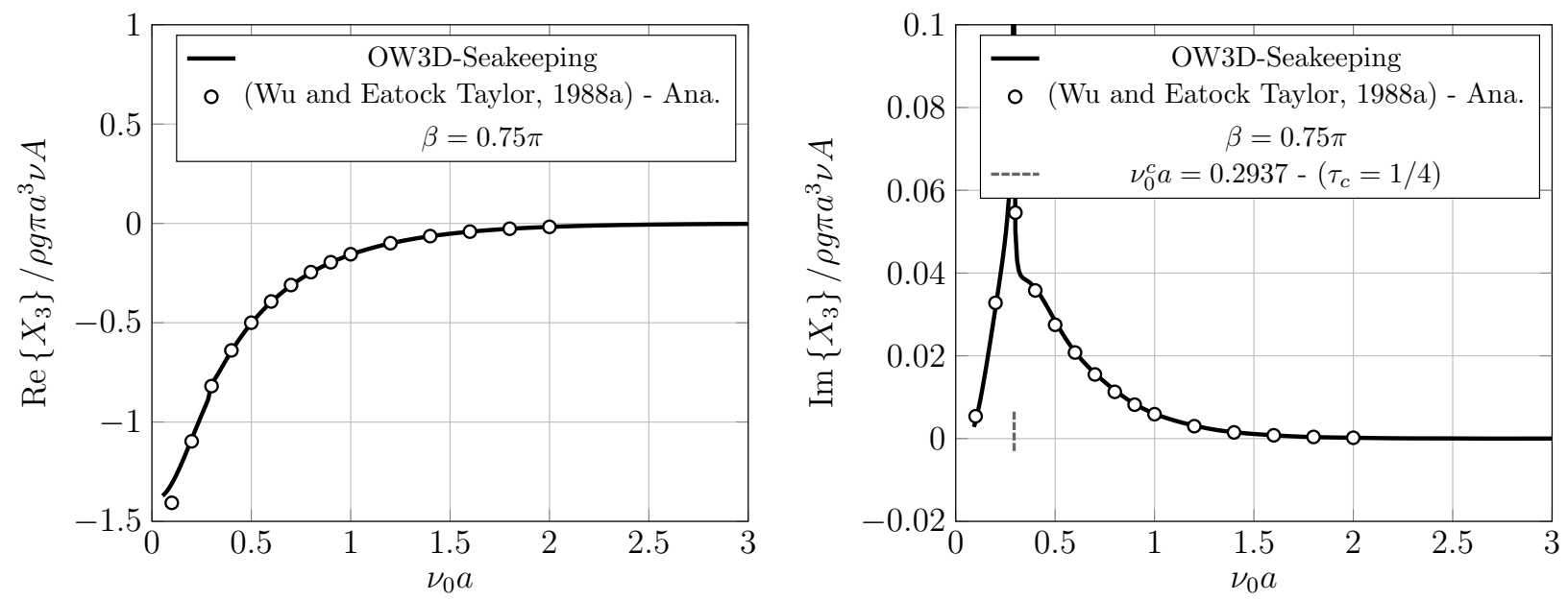

Figure 16: The wave excitation force in heave , $X_{3}$, on the submerged sphere $\beta=3 \pi / 4, F r=0.40$ 

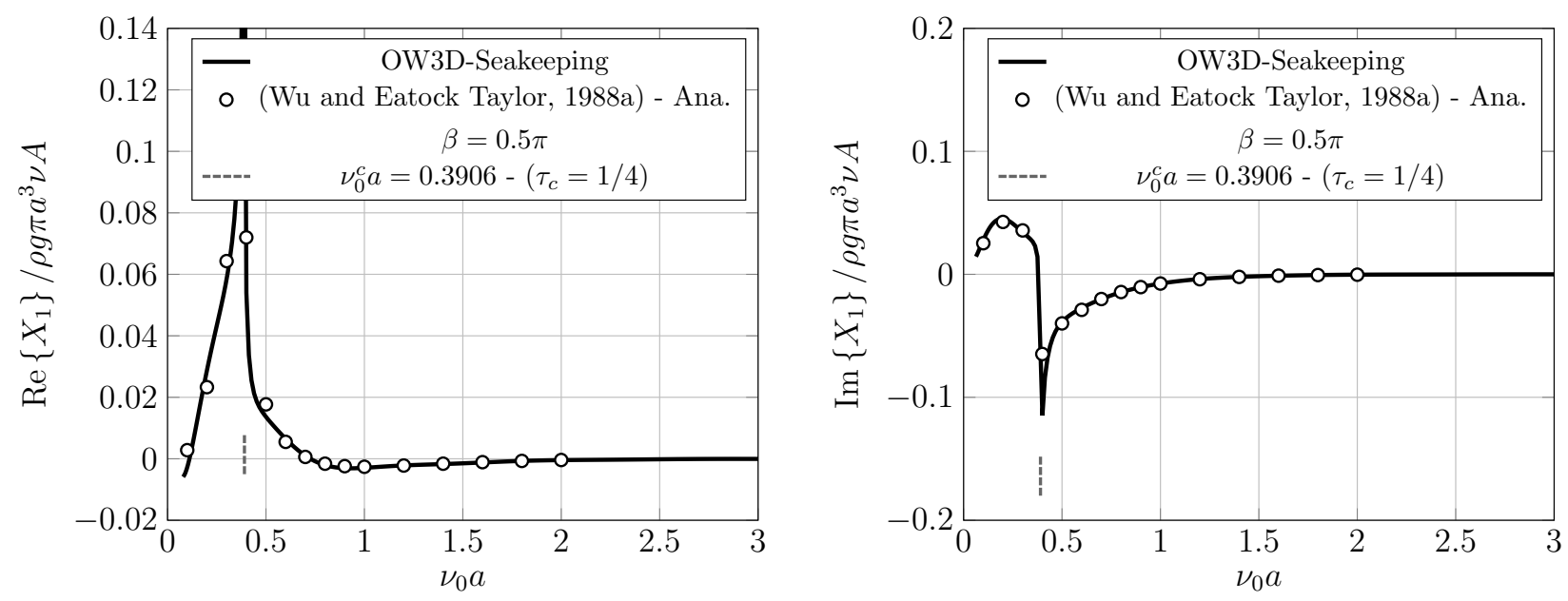

Figure 17: The wave excitation force in surge,$X_{1}$, on the submerged sphere, $\beta=\pi / 2, F r=0.40$
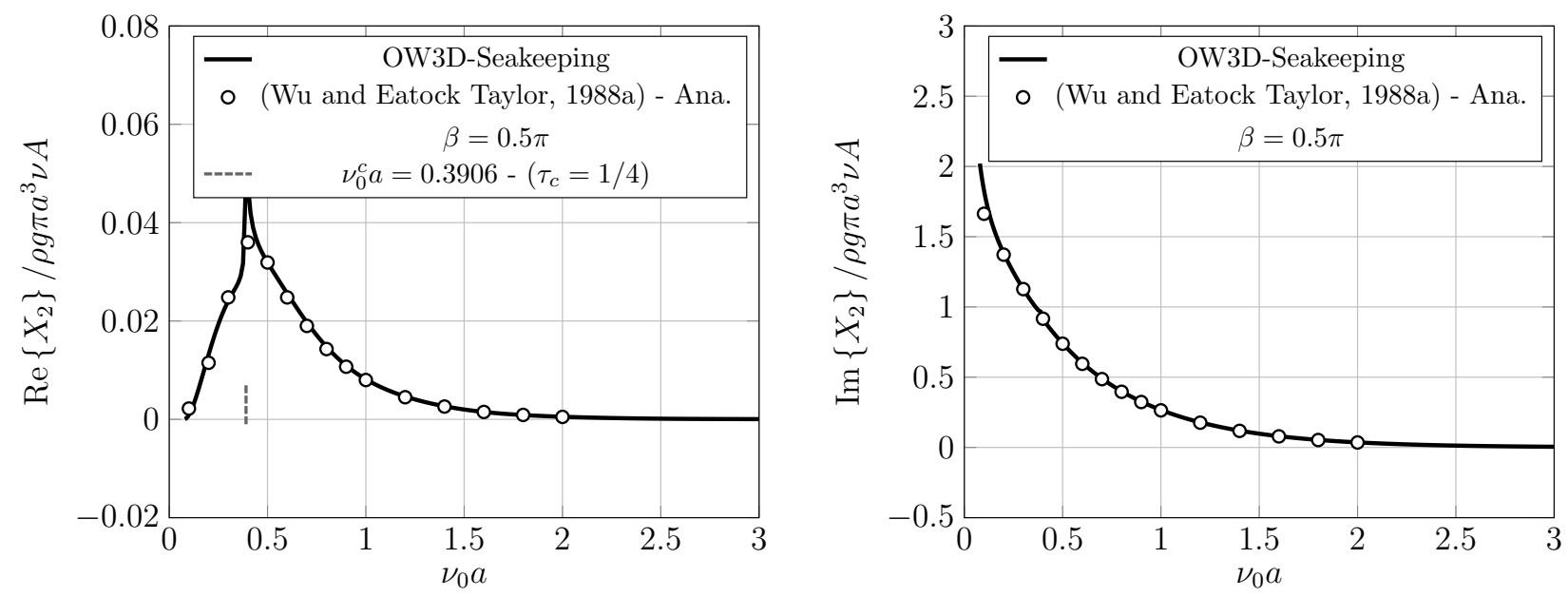

Figure 18: The wave excitation force in sway,$X_{2}$, on the submerged sphere, $\beta=\pi / 2, F r=0.40$
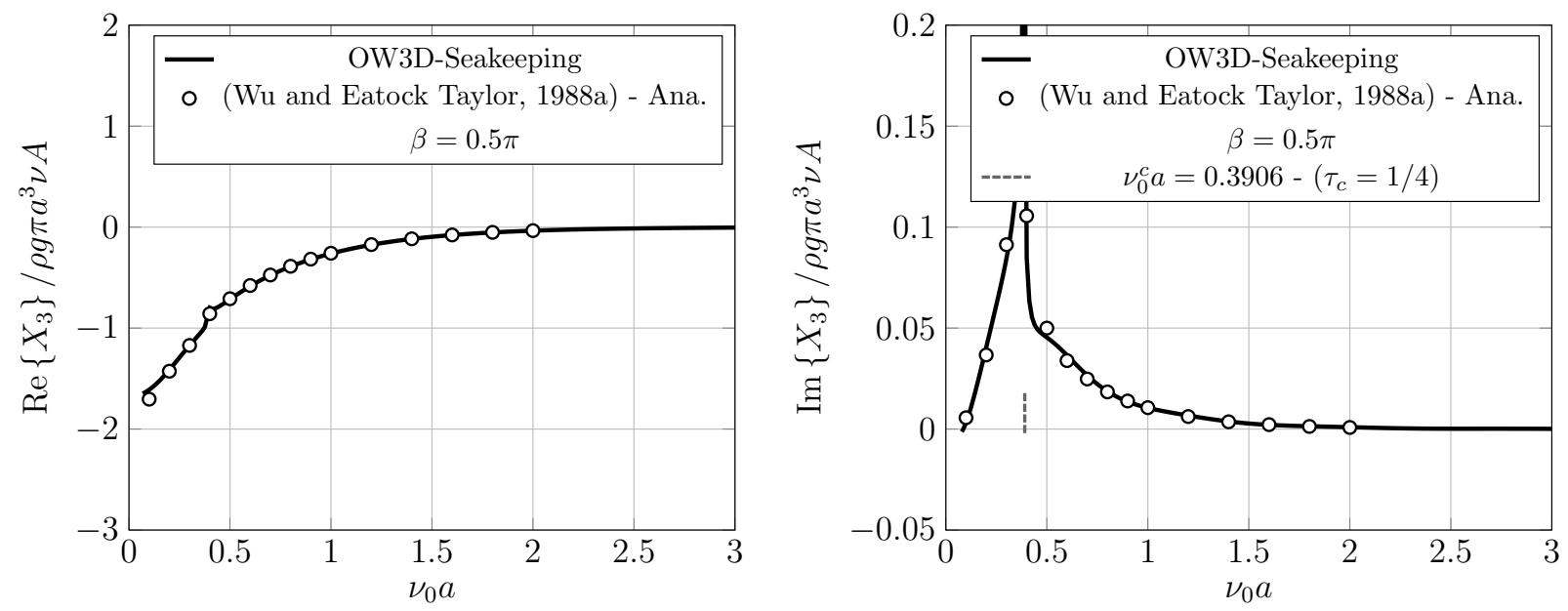

Figure 19: The wave excitation force in heave,$X_{3}$, on the submerged sphere, $\beta=\pi / 2, F r=0.40$ 

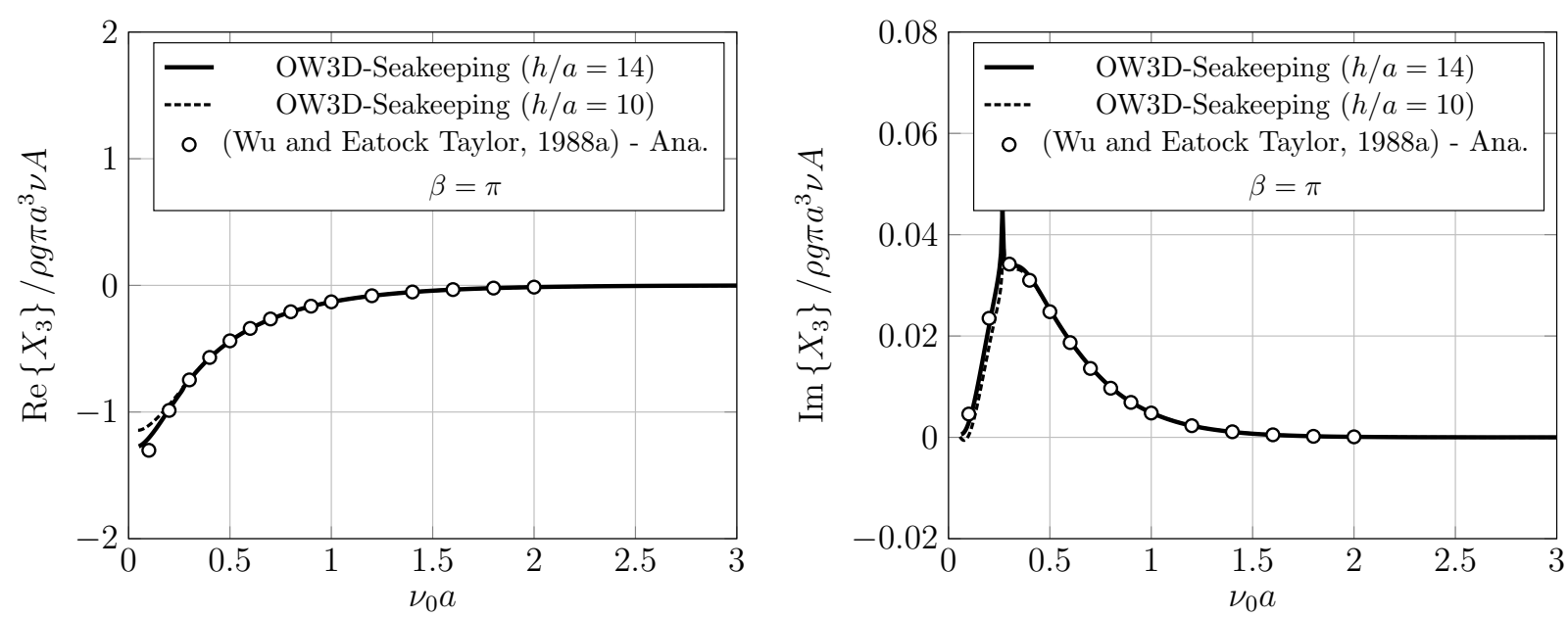

Figure 20: An example showing the effect of the depth of the computational domain $h$ on the numerical results for the wave excitation forces which are for the deep-water condition. The results are for the heave force on the submerged sphere with $\beta=\pi$ and $F r=0.4$

\subsubsection{Discussion of the results}

The wave excitation forces for the circular cylinder both in following seas and in head seas are compared with the reference numerical solution. A reasonable agreement can be observed between the solutions especially for the surge forces. An exact match has not been obtained, which may be related to the fact that the double-body flow is used instead of the steady wave resistance solution for $\phi_{b}^{\prime}$. It is known the difference between these two base flows is more pronounced at high Froude numbers.

The comparison has also been made between the numerical results and the analytical solutions for the wave forces applied on the moving submerged sphere. Very good agreement is shown except for very long waves (small $\nu_{0} a$ ). This is due to the finite dimension of the computational domain along the $z$ direction, where the numerical results can not agree very well with the analytical solutions, which are in fact for the deep-water condition. The results in this range can be improved by increasing the computational depth, as is shown in Figure 20.

Moreover it is interesting to mention that in the case $\beta=\pi / 2$, the wave excitation force along the $x$ axis $\left(X_{1}\right)$ is not zero, as can be seen from Figure 17. This is however expected since the forward speed of the body disturbs the otherwise symmetric pattern of the scattered waves along the $y$ axis. Note that this symmetry is preserved and the surge force will be zero if the sphere has no forward speed. 


\subsection{Bulk carrier}

In the developed seakeeping code, the 3D computational grid for any arbitrary geometry (including ship hulls), can be built using the Hyperbolic Grid Generation technique [39]. According to this method, first a surface grid is grown over a desired part of the geometry. Afterwards, the resulting surface grid is marched into the fluid to create a volume grid. During these stages, a set of boundary conditions should also be defined in order for the surface and the volume grids to be generated correctly. The final overlapping grid is then obtained by combining all these volume grids. This grid generation method is provided by the open-source grid generator Ogen [40].

An overlapping grid for a bulk carrier ship hull is generated using this tool. The geometry of this ship, which is called RIOS bulk carrier, is from [41]. Part of the final overlapping grid for the ship hull can be seen in Figures 21 - 24. The depth of the computational grid is $h=2 L_{p p}$, and the background cylindrical grid has a radius of $R_{c}=18 L_{p p}$. This hull geometry has been represented by 3 volume grids. Two of them are assigned for the regions close to the bow and the stern. The rest of the hull is covered by the third volume grid. In order to get the ghost layer generated properly at the waterline, it is necessary to have an almost wall-sided hull at that elevation. This can also be observed from Figure 21 towards the stern region of the hull. Similar to the cylinder and the sphere cases shown before, the generated grid for the ship hull is placed inside a cylindrical background grid, see also Figure 10. In addition, very strong grid stretching over the free surface is employed. Consequently the grid spacing close to the far-field truncation boundary $S_{\infty}$, is at least 2 to 3 times the length of the body. Using this strategy, combined with the limited frequency-content of the pseudo-impulsive forcing, ensures that there will be negligible reflection from $S_{\infty}$ during the limited duration of the calculation.

The forward-speed diffraction problem is also solved for the bulk carrier. The ship has a Froude number $F r=U / \sqrt{g L_{p p}}=0.18$, where $L_{p p}$ is the length between the perpendiculars. Both head seas and following seas will be considered. 


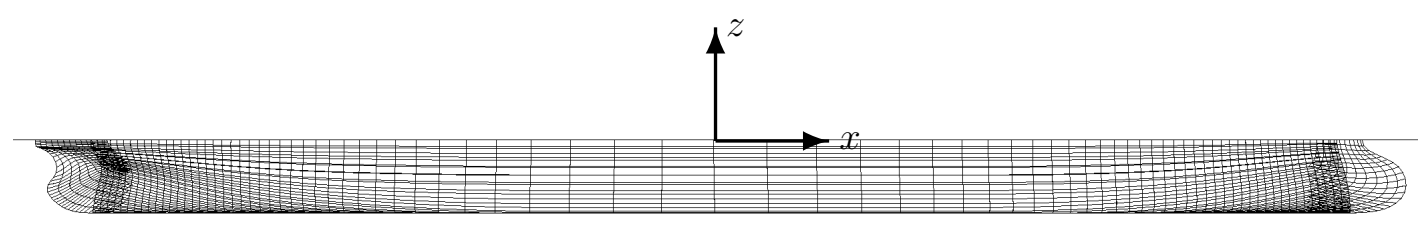

Figure 21: Part of the overlapping grid for the RIOS bulk carrier (side view)

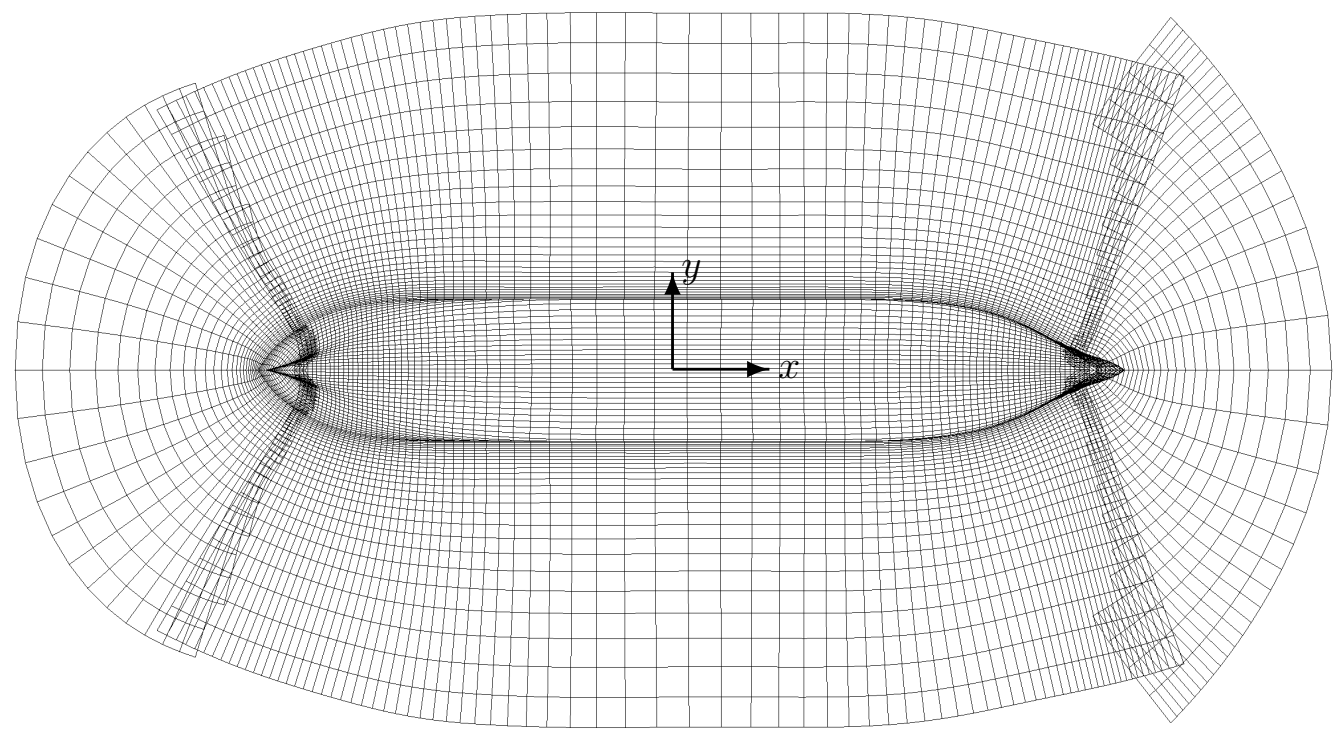

Figure 22: Part of the overlapping grid for the RIOS bulk carrier (top view)

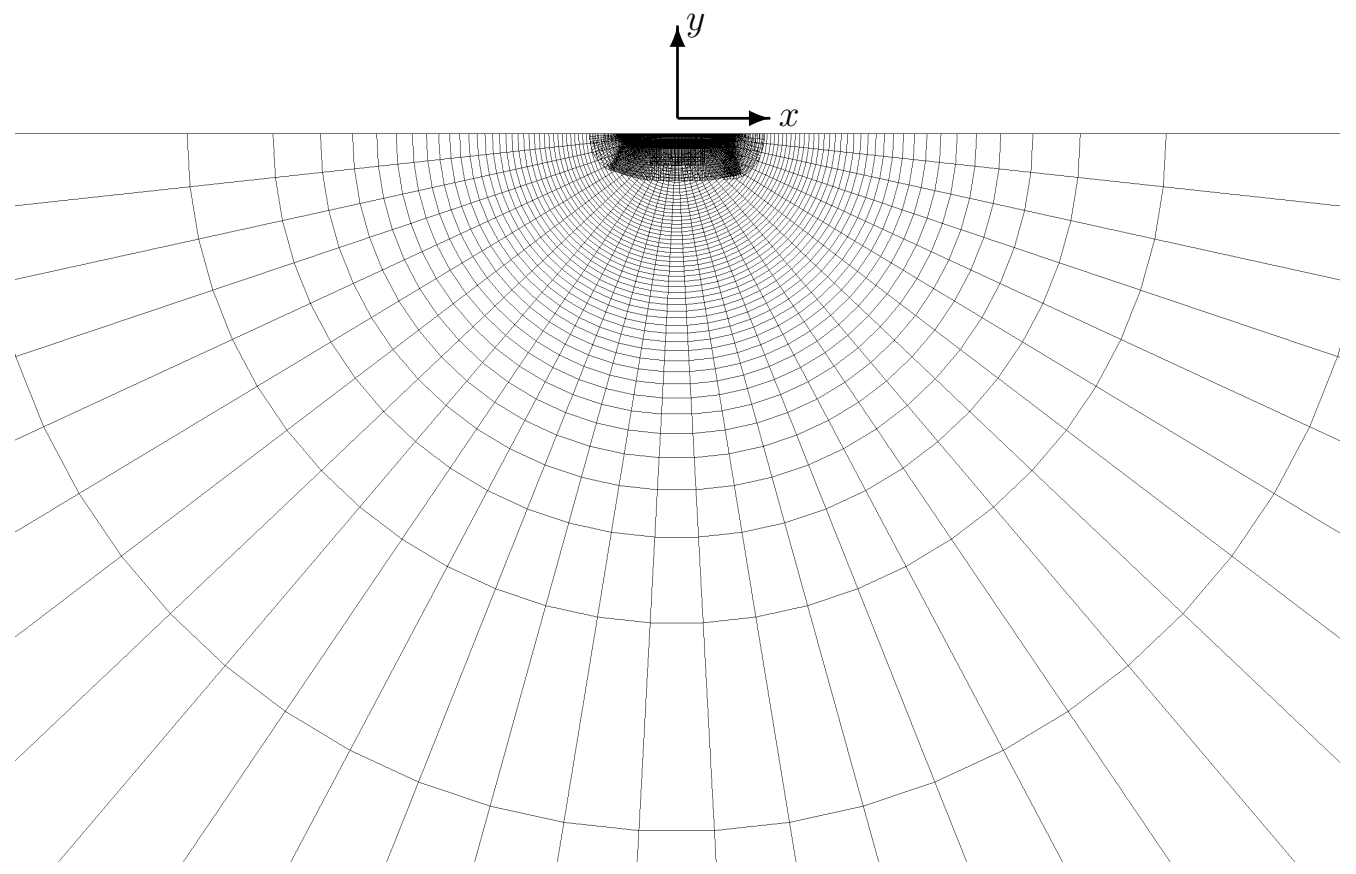

Figure 23: Part of the overlapping grid for the RIOS bulk carrier, shown together with the cylindrical background grid (top view). Note that the $y$-coordinate of the origin is shifted for better illustration. Moreover due to symmetry, only half of the geometry is used for the numerical calculation. 


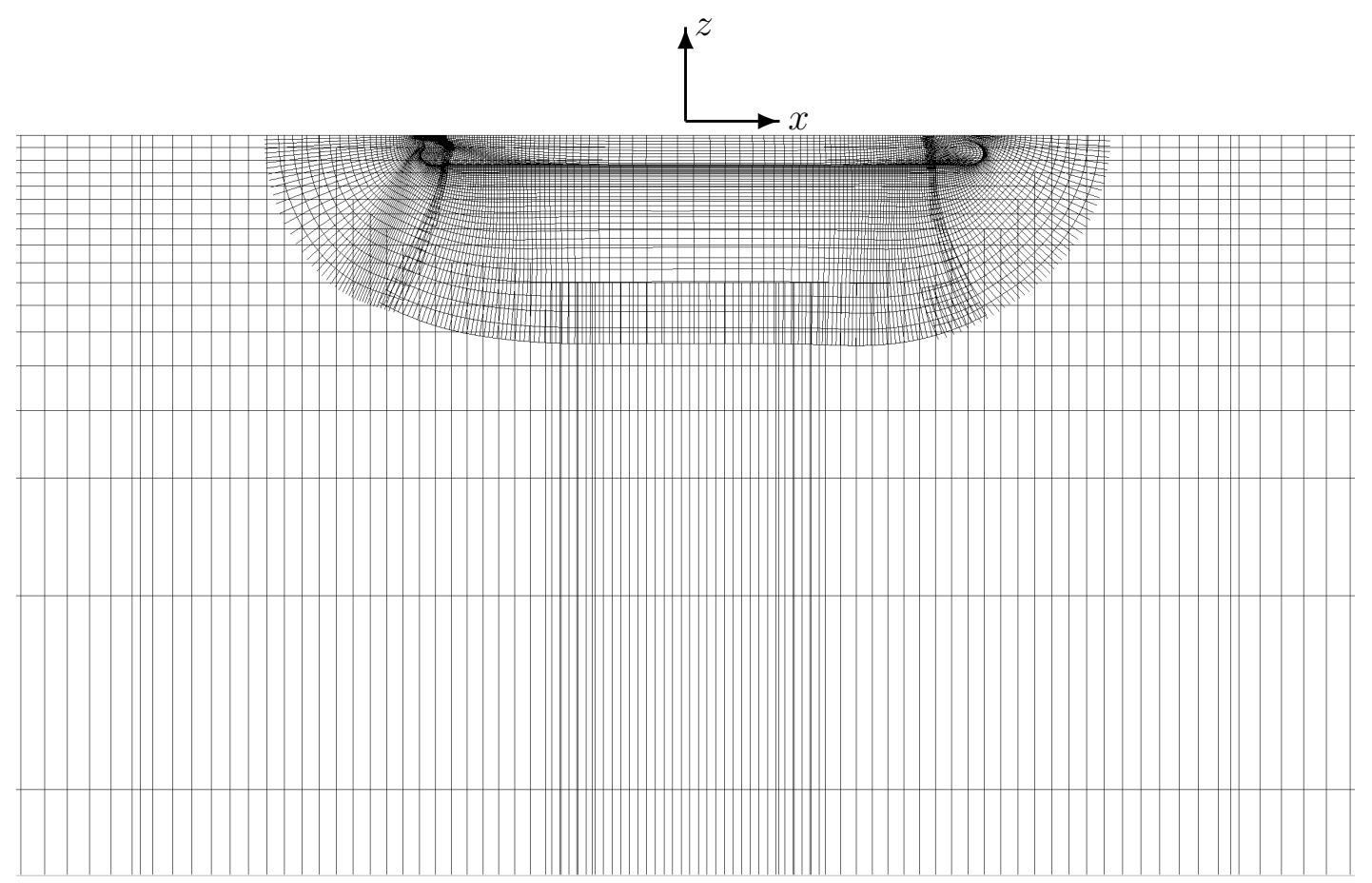

Figure 24: Part of the overlapping grid for the RIOS bulk carrier, shown together with the cylindrical background grid (side view). Note that the $z$-coordinate of the origin is shifted for better illustration.

\subsubsection{Head seas $(\beta=\pi)$}

The calculated scattering forces resulting from the time-domain simulation together with the corresponding pseudo-impulsive incident wave elevation $\zeta_{0}(t)$ are shown in Figure 25. The frequency-domain results for the scattering forces are computed using the Fourier transformation in (2.50). The total wave excitation forces in the frequency domain (including the magnitude and also the phase angle) are presented in Figures 26-28. In the plots, the breadth of the ship is denoted by $B$, and $\theta_{0}$ is the phase angle of the computed result in radians. Note also that the numerical results for the wave excitation forces are given for both the Neumann-Kelvin and the double-body linearizations. Comparison is made with the measurements by [41], and also with the numerical solutions obtained by the timedomain boundary element code TiMIT [42], which is based on the Neumann-Kelvin linearization. The hydrodynamic coefficients, including added mass and damping have already been calculated using this solver for the bulk carrier [26], and the frequency-domain results for the ship motions (RAOs) are presented in Figures 29-31. In these figures, comparison is made to experimental data [41] and the results from TiMIT. The wave number is denoted by $k$ in the plots. Note that the critical frequency in this case is equivalent to $\lambda_{c} / L=4.71378$. 

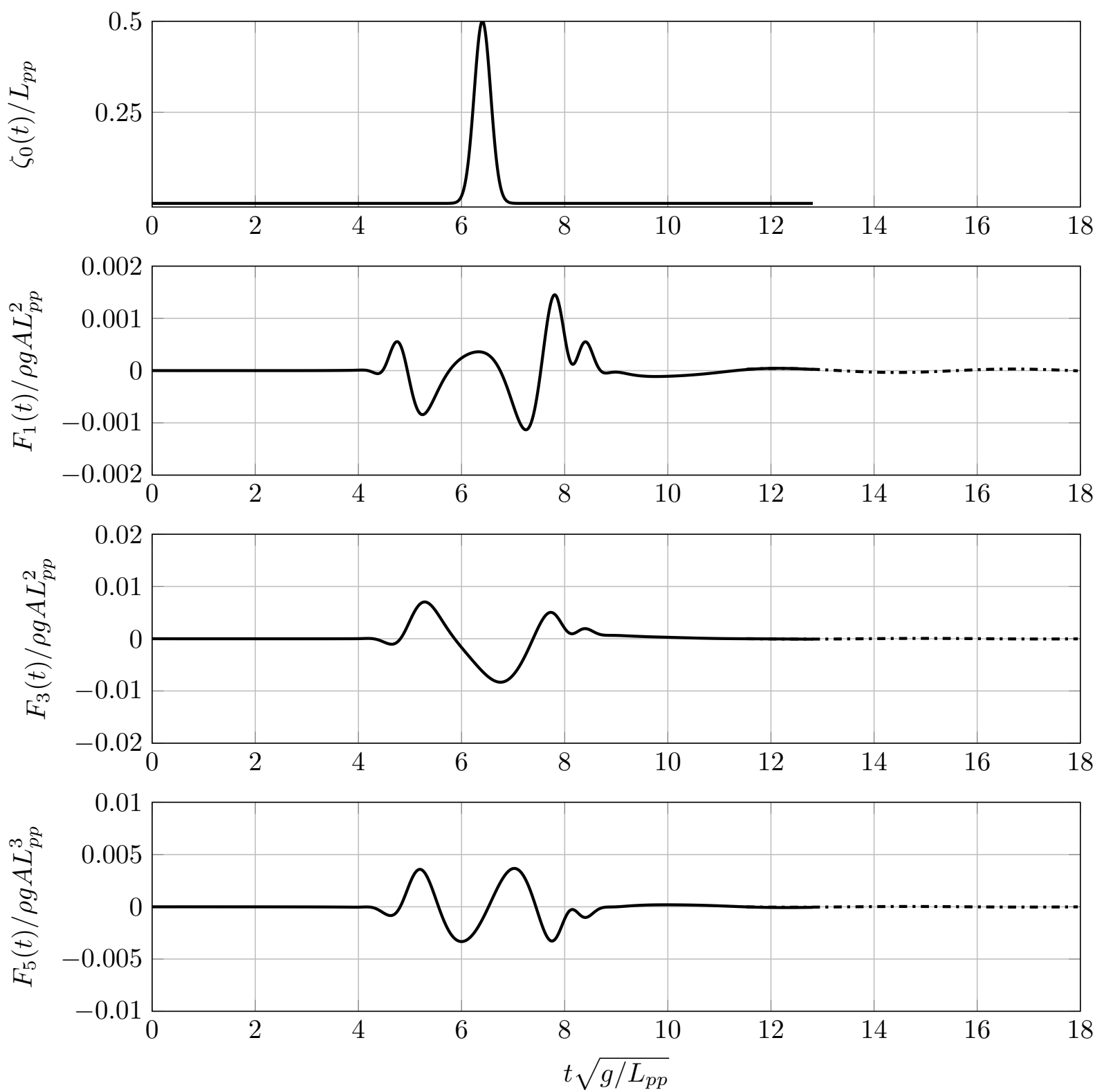

Figure 25: The pseudo-impulsive incident wave elevation $\zeta_{0}(t)$ given by $(2.24)$, and the time-domain scattering wave forces in the surge, heave and pitch directions for the bulk carrier, $\beta=\pi$ and $F r=0.18$. The asymptotic extrapolation at the critical frequency is also shown for each direction by the dashdotted line. 

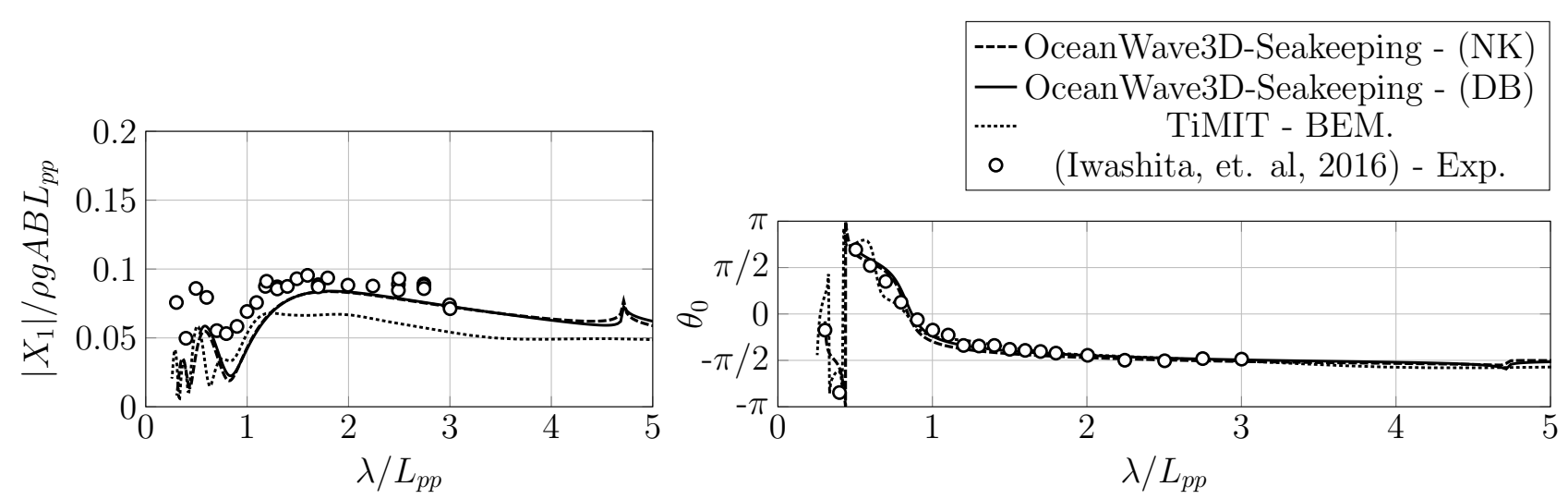

Figure 26: The wave excitation force in surge,$X_{1}$, for the bulk carrier, $\operatorname{Fr}=0.18$ and $\beta=\pi$
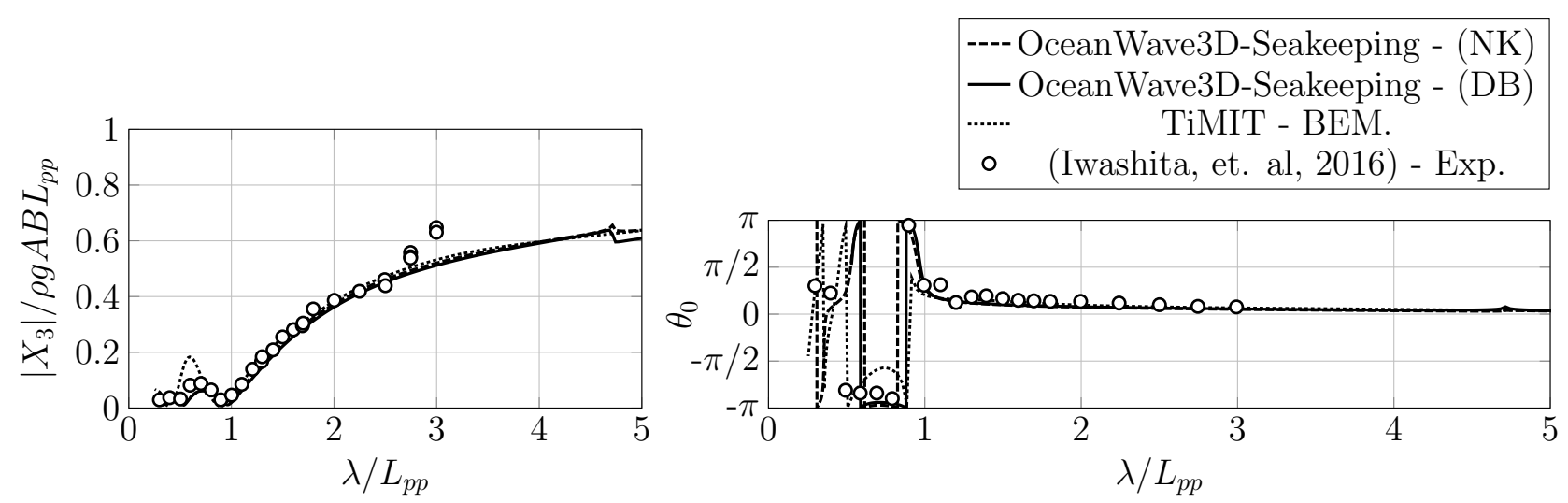

Figure 27: The wave excitation force in heave , $X_{3}$, for the bulk carrier, Fr $=0.18$ and $\beta=\pi$
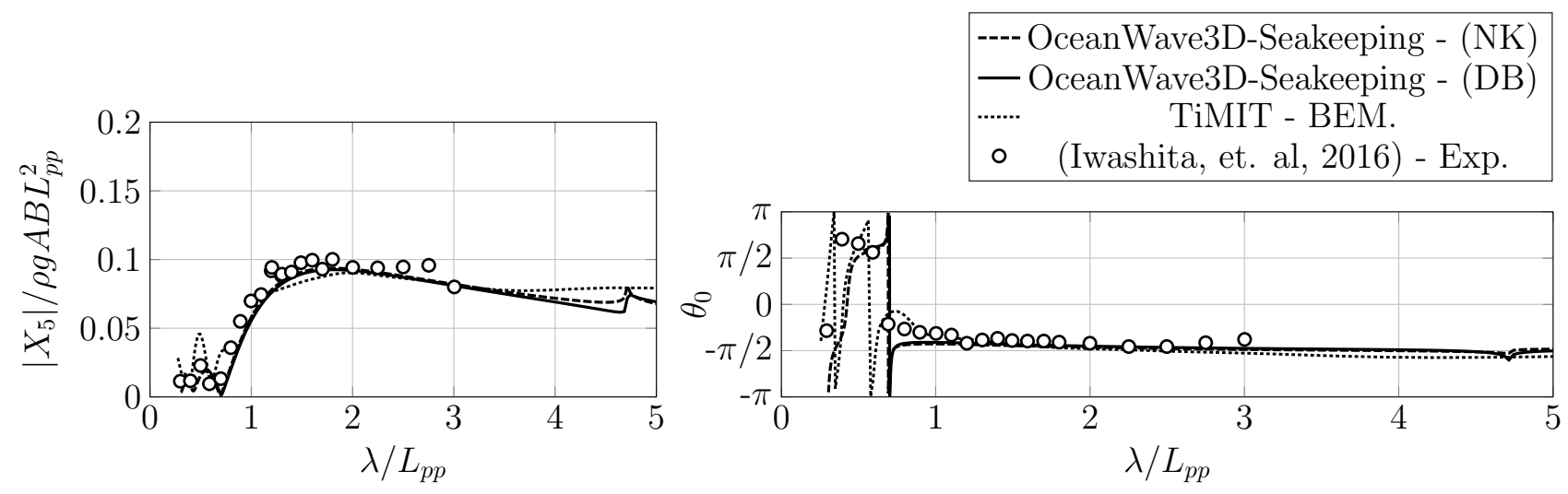

Figure 28: The wave excitation force in pitch , $X_{5}$, for the bulk carrier, $\operatorname{Fr}=0.18$ and $\beta=\pi$ 

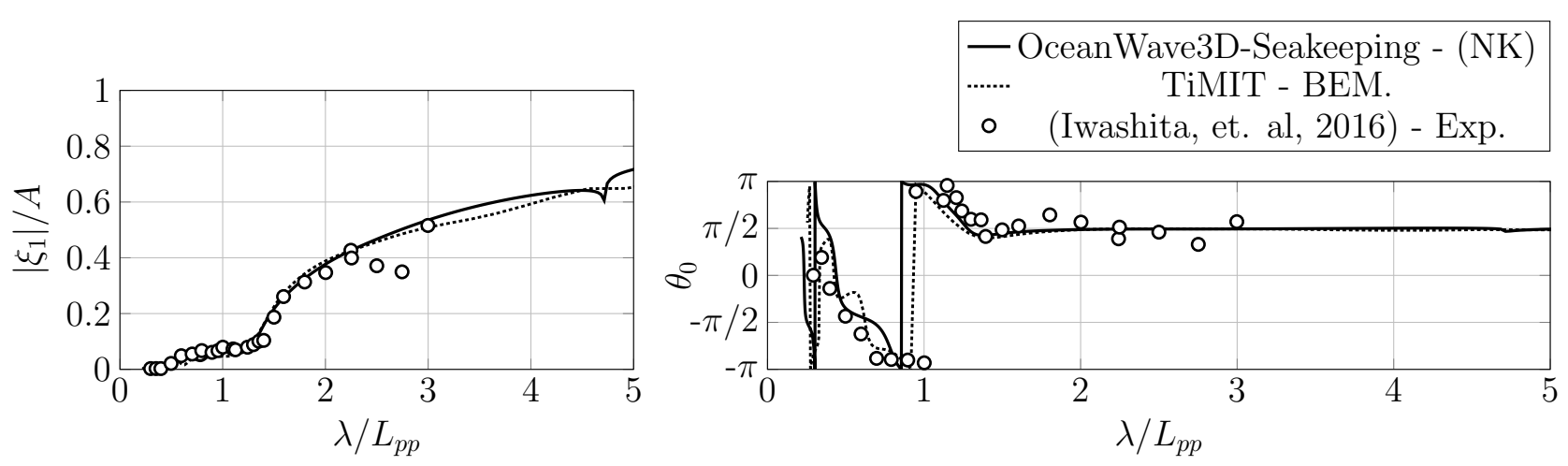

Figure 29: The motion response in surge,$\xi_{1}$, for the bulk carrier, $\operatorname{Fr}=0.18$ and $\beta=\pi$
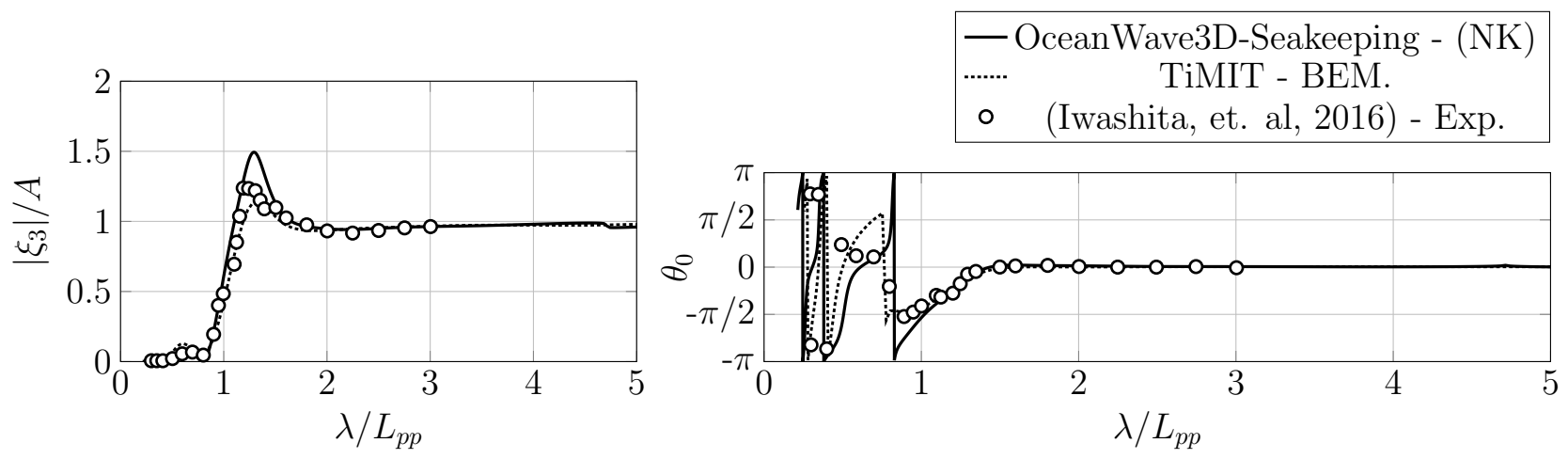

Figure 30: The motion response in heave,$\xi_{3}$, for the bulk carrier, $\mathrm{Fr}=0.18$ and $\beta=\pi$
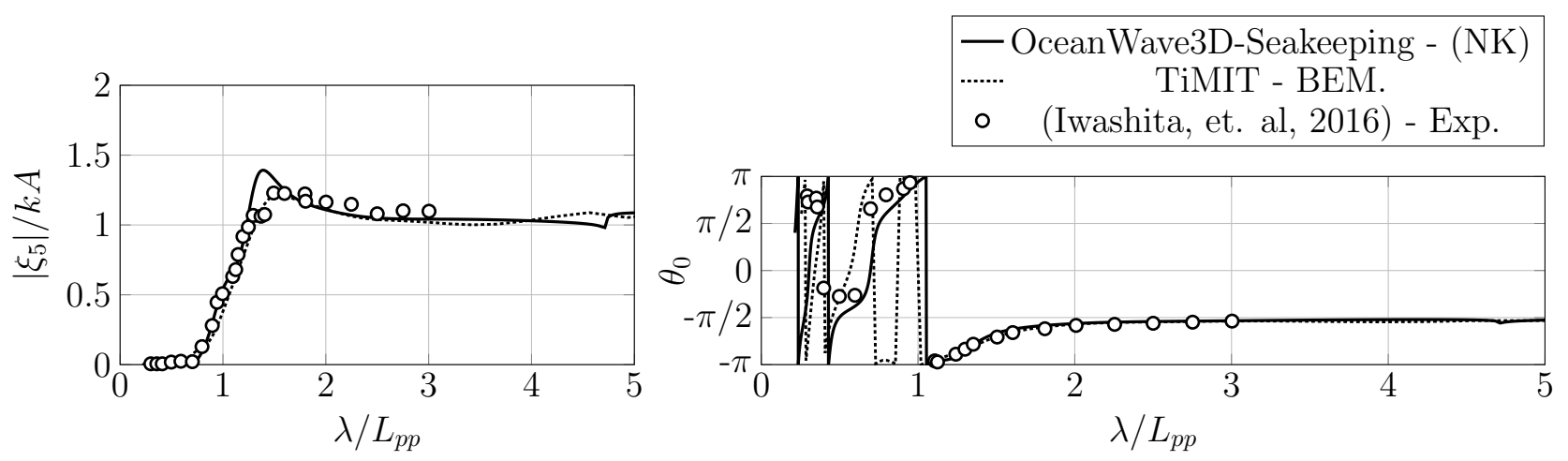

Figure 31: The motion response in pitch,$\xi_{5}$, for the bulk carrier, $\operatorname{Fr}=0.18$ and $\beta=\pi$ 


\subsubsection{Following seas $(\beta=0)$}

The wave excitation forces and the ship motions are also calculated for the following-seas condition. As was mentioned earlier, it is required to solve 3 separate diffraction problems to obtain the frequencydomain solutions in the $\omega_{01}, \omega_{02}$ and $\omega_{03}$ regions. The results for the time-domain scattering forces in the surge, heave and pitch directions are shown in Figures $32-34$. The relation between the encounter frequency $\omega_{e}$, the wave frequency $\omega_{0}$ and $\lambda / L_{p p}$ for these simulations are also plotted in Figure 35. The end of the $\omega_{03}$ region is at $\lambda / L=0.2036$ which is marked by a triangle. The end of $\omega_{02}$ is also marked by a filled triangle at $\lambda / L=0.8143$. It can be seen that the main part of the solution in the frequency domain falls in fact inside the $\omega_{01}$ region. The critical frequency is also equivalent to $\lambda_{c} / L=0.8143$ and $\lambda_{c} / L=0.1397$.

The frequency-domain wave excitation forces are presented in Figures 36-38. These results are compared with the boundary element solution from TiMIT, and also to the numerical results by [41]. The results from [41] are reproduced using the digitization of the original plots. In addition, the results for the ship motions (RAOs) are compared with the measurements from [41] and with TiMIT in Figure 39-41. 

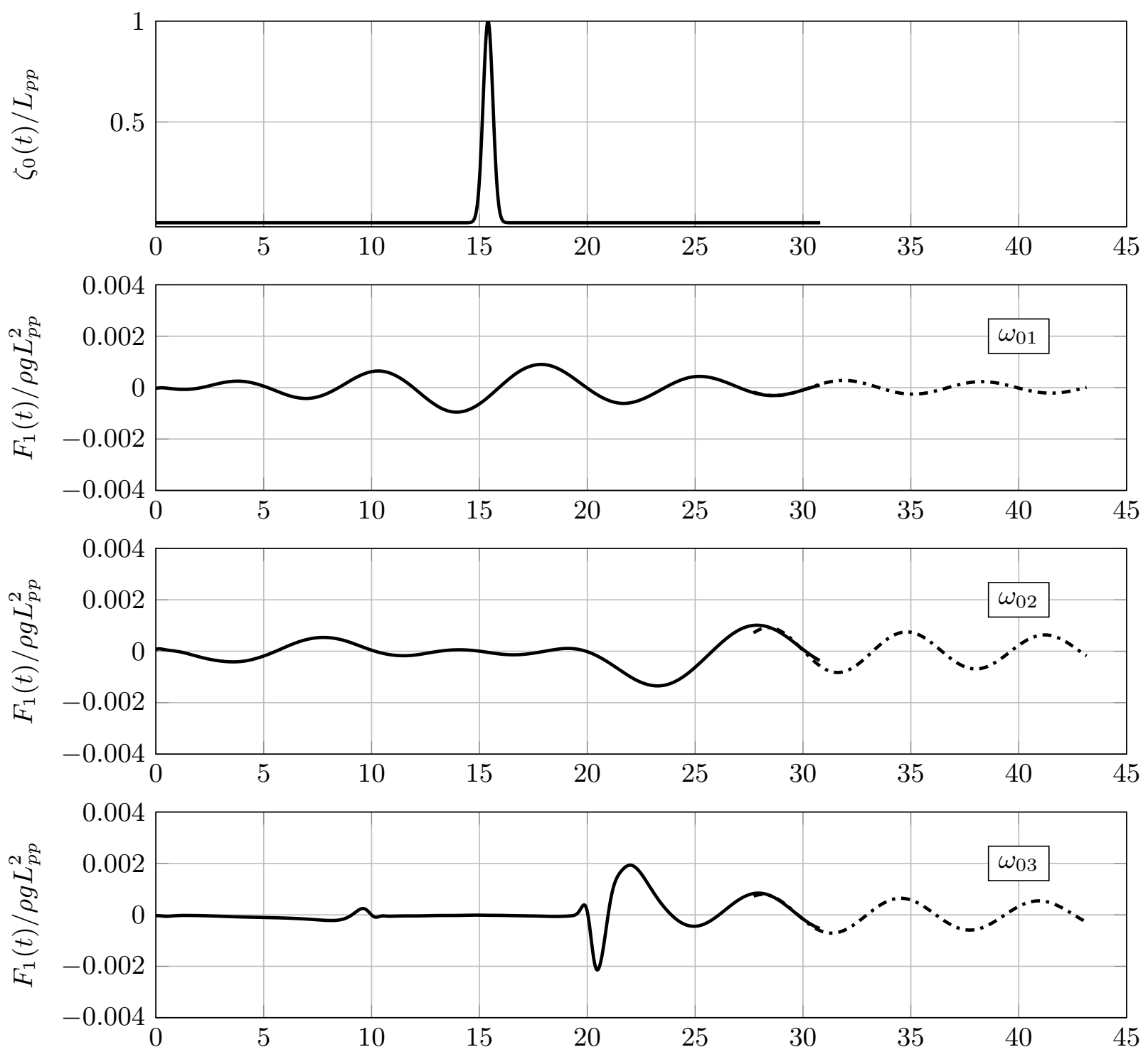

Figure 32: The pseudo-impulsive incident wave elevation and the time-domain scattering forces in the surge direction for the bulk carrier in the case of following seas $(\beta=0)$. Note that 3 time-domain plots are shown which are the forces corresponding to the $\omega_{01}, \omega_{02}$ and $\omega_{03}$ regions in the frequency domain. The asymptotic extrapolation at the critical frequency is also shown for each force by the dash-dotted line. 

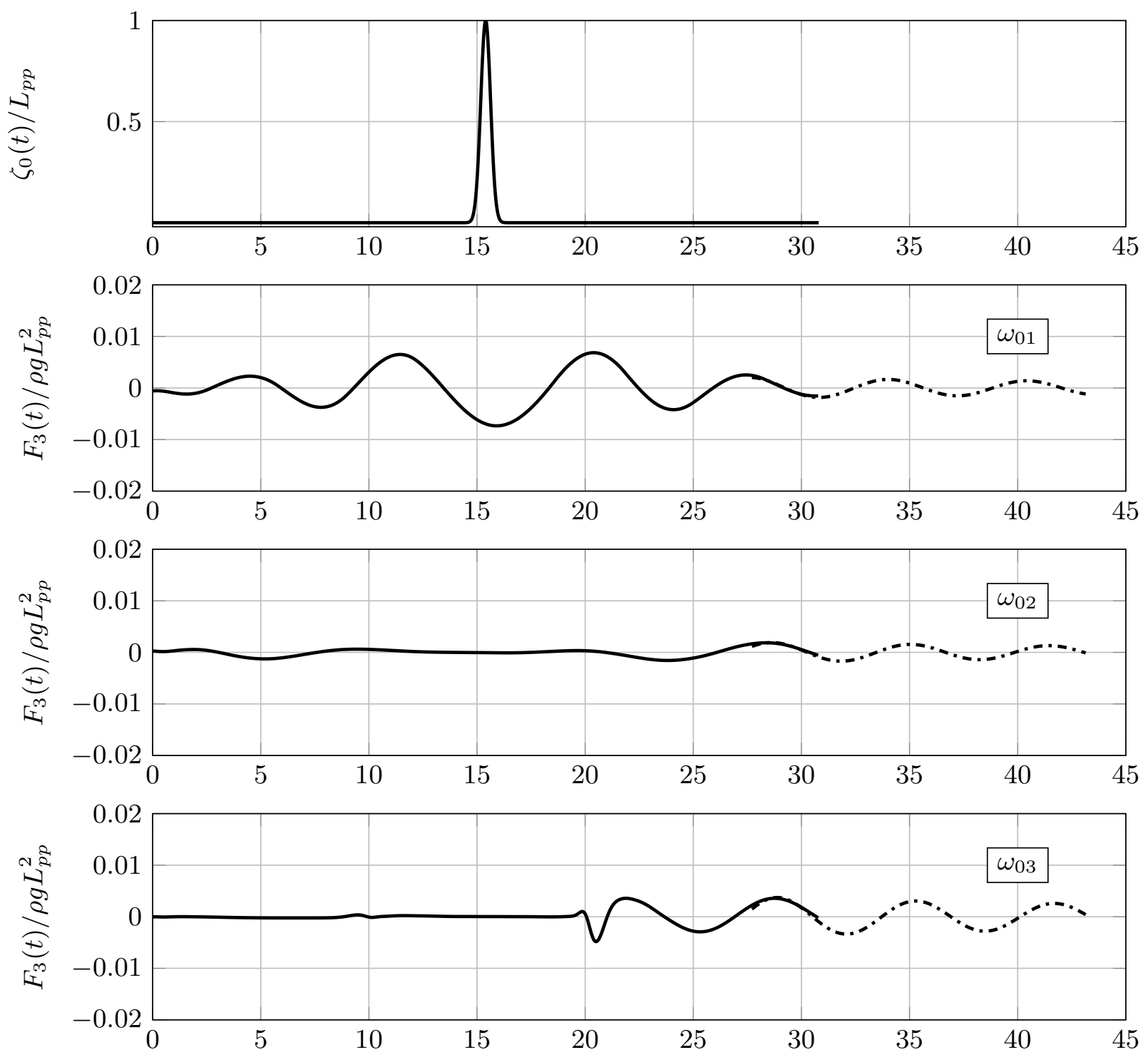

Figure 33: The pseudo-impulsive incident wave elevation and the time-domain scattering forces in the heave direction for the bulk carrier in the case of following seas $(\beta=0)$. Note that 3 time-domain plots are shown which are forces corresponding to the $\omega_{01}, \omega_{02}$ and $\omega_{03}$ regions in the frequency domain. The asymptotic extrapolation at the critical frequency is also shown for each force by the dash-dotted line. 

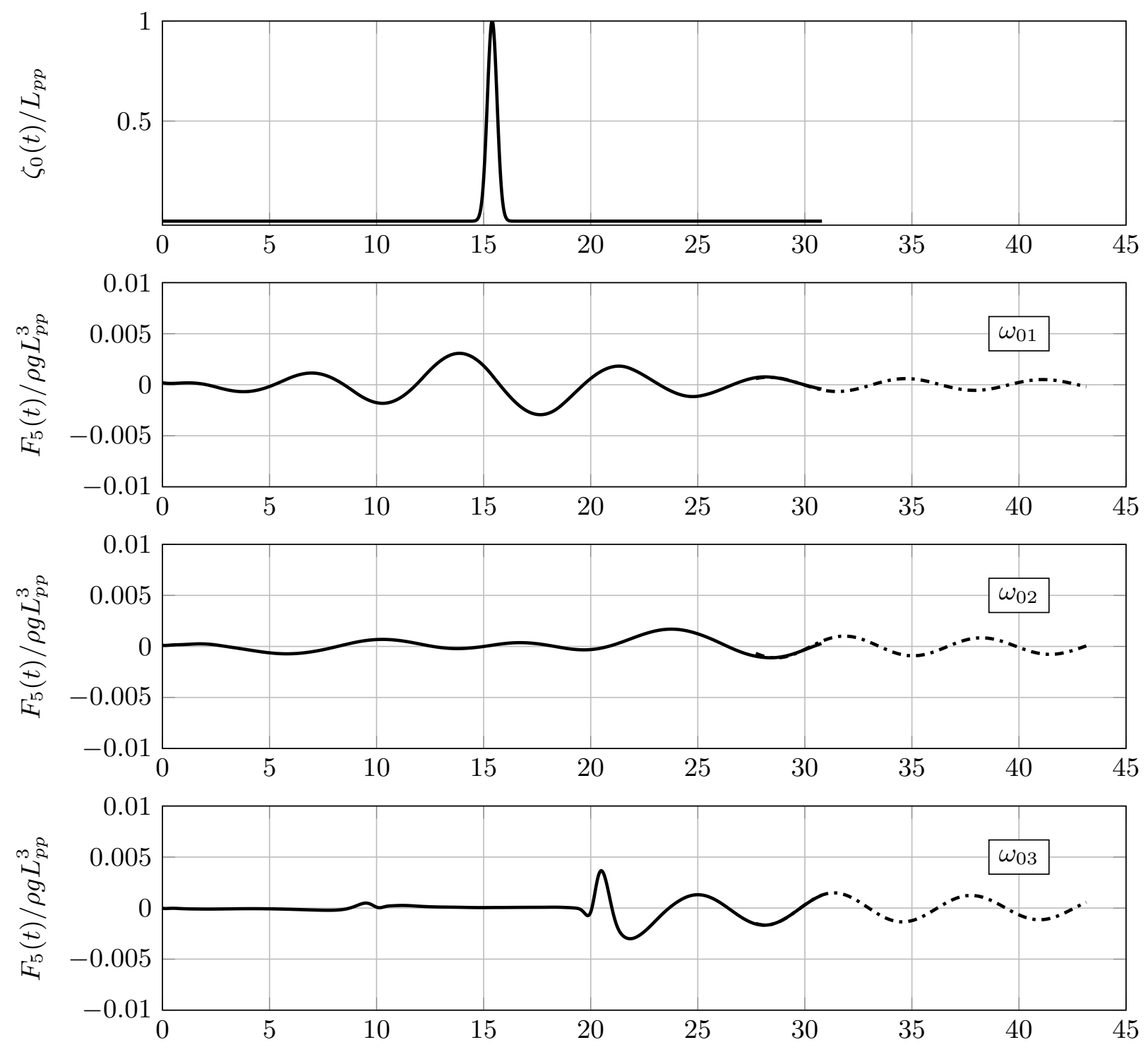

Figure 34: The pseudo-impulsive incident wave elevation and the time-domain scattering forces in the pitch direction for the bulk carrier in the case of following seas $(\beta=0)$. Note that 3 time-domain plots are shown which are the force corresponding to the $\omega_{01}, \omega_{02}$ and $\omega_{03}$ regions in the frequency domain. The asymptotic extrapolation at the critical frequency is also shown for each force by the dash-dotted line. 

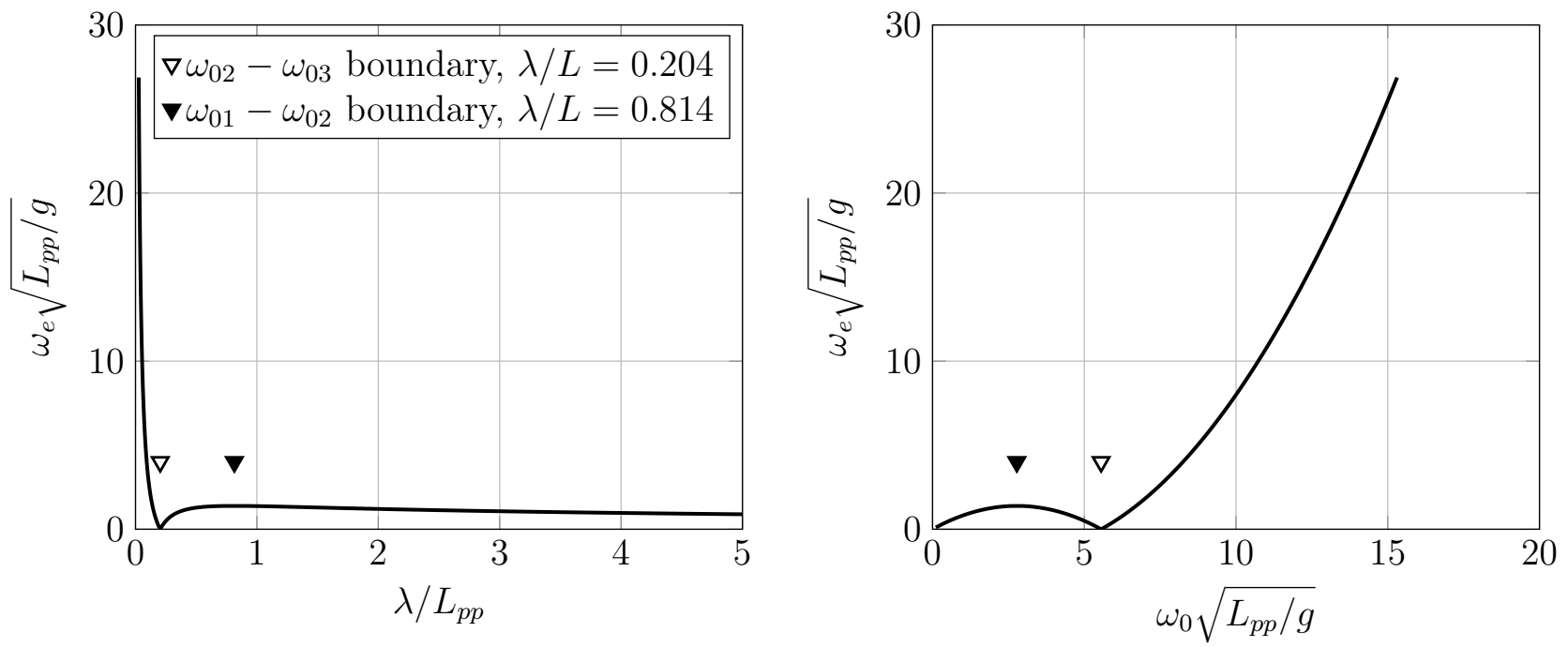

Figure 35: The relation between the encounter frequency, the wave frequency and $\lambda / L_{p p}$, for the case of the bulk carrier in the following seas with $F r=0.18$ 

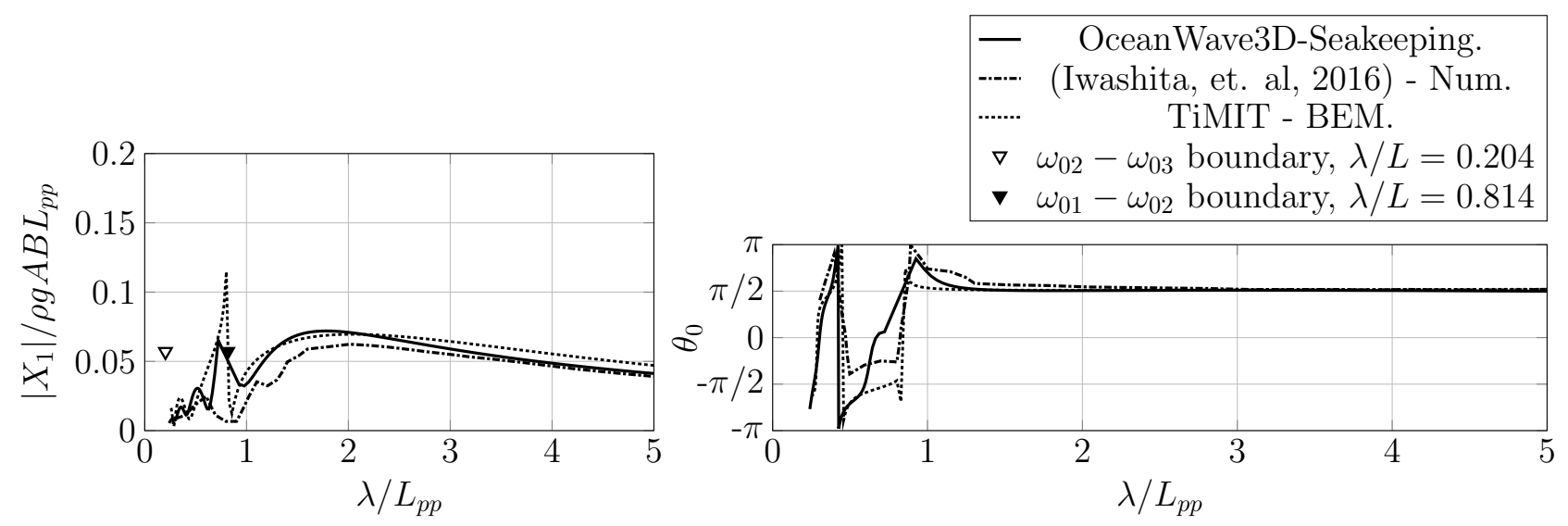

Figure 36: The wave excitation force in surge , $X_{1}$, for the bulk carrier, $\mathrm{Fr}=0.18$ and $\beta=0$

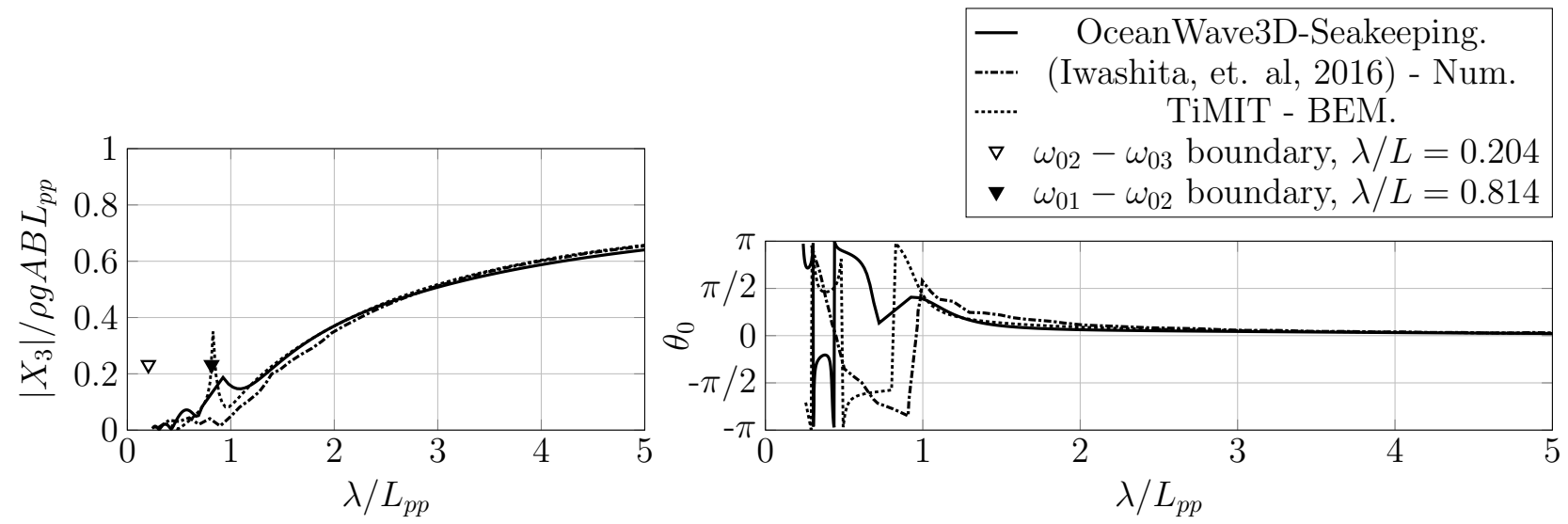

Figure 37: The wave excitation force in heave,$X_{3}$, for the bulk carrier, $\operatorname{Fr}=0.18$ and $\beta=0$

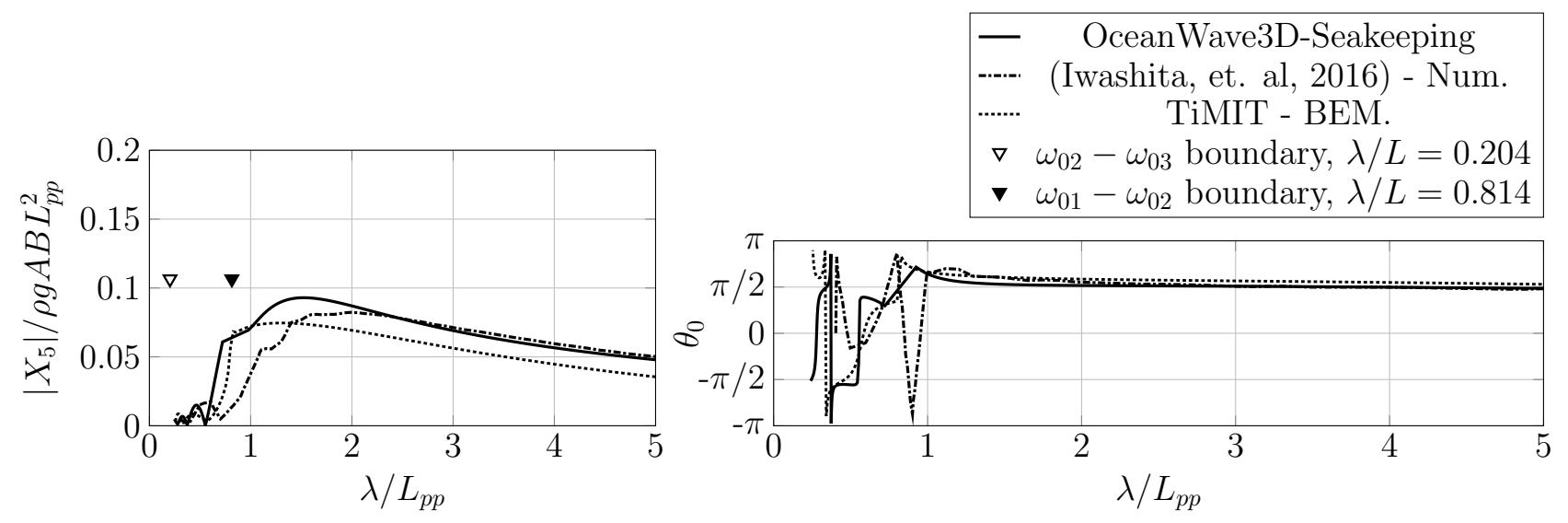

Figure 38: The wave excitation force in pitch,$X_{5}$, for the bulk carrier, $\mathrm{Fr}=0.18$ and $\beta=0$ 

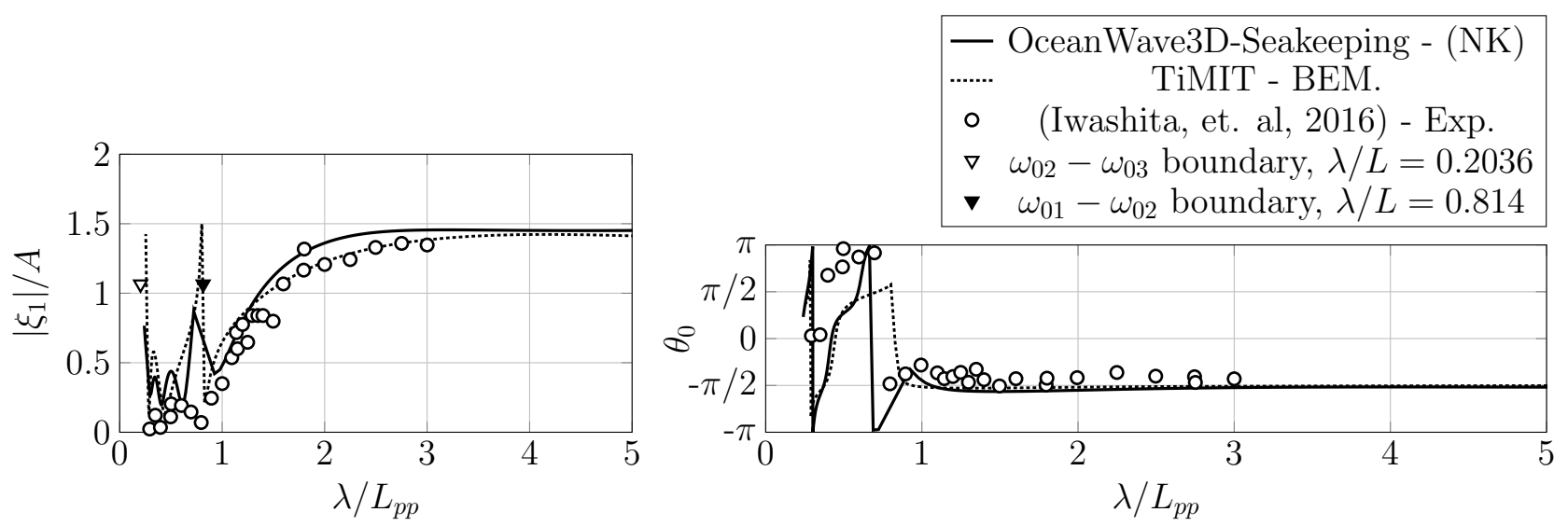

Figure 39: The motion response in surge,$\xi_{1}$, for the bulk carrier, $\operatorname{Fr}=0.18$ and $\beta=0$
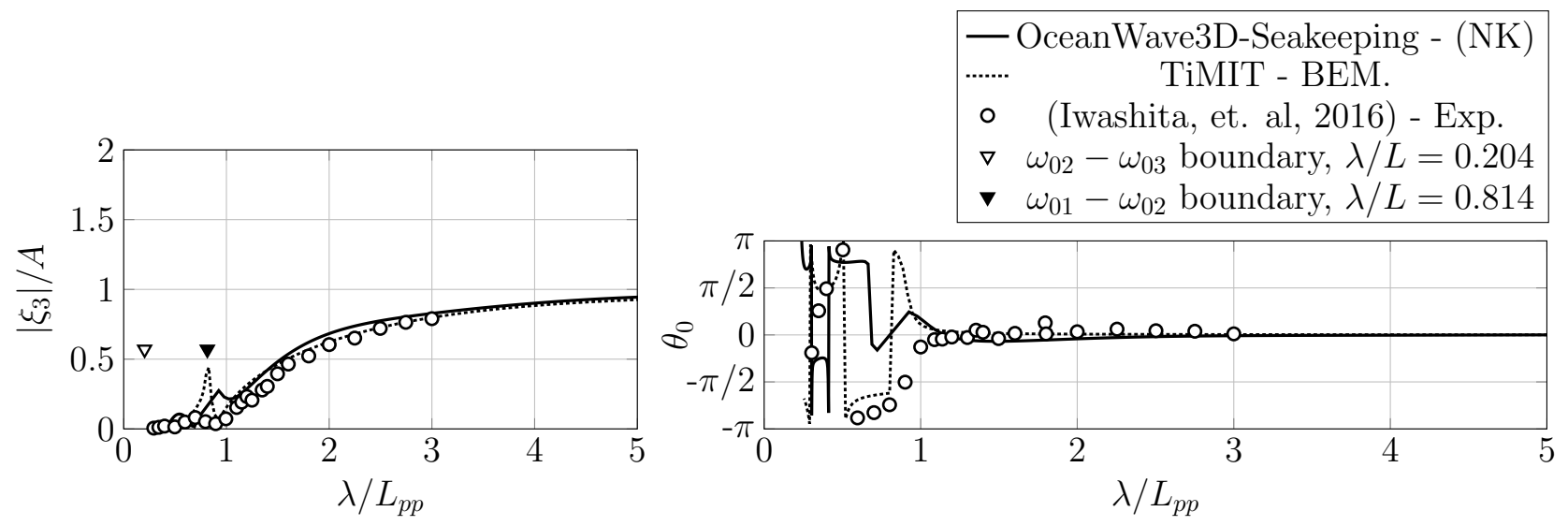

Figure 40: The motion response in heave,$\xi_{3}$, for the bulk carrier, $\mathrm{Fr}=0.18$ and $\beta=0$
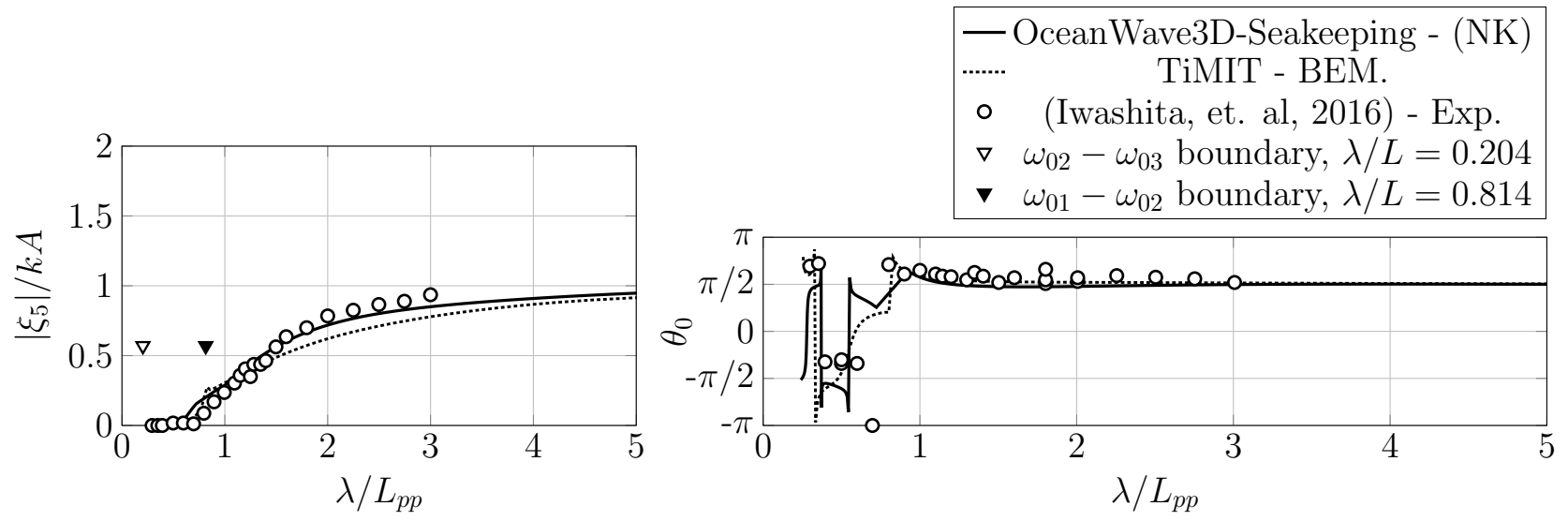

Figure 41: The motion response in pitch,$\xi_{5}$, for the bulk carrier, $\operatorname{Fr}=0.18$ and $\beta=0$ 


\subsubsection{Discussion of the results}

The calculated wave forces in the head-seas condition, show a very good agreement, both with the measurements and the TiMIT calculations. The results based on the Neumann-Kelvin and the double-body linearization for this geometry are very similar, except for the surge and pitch forces in very long waves. The calculated motions of the body (in both head seas and following seas), including the amplitude and the phase, also agree reasonably well with the measurements and with the TiMIT results.

For the following-seas case, an acceptable agreement has also been achieved between the excitation forces obtained by the developed solver, and those calculated by other numerical seakeeping codes. The phase of the wave excitation forces calculated by OW3D and TiMIT are in better agreement. Note also that the diffraction solution in the $\omega_{01}$ region extends over a larger range of $\lambda / L_{p p}$. The expected behavior of the phasing of the wave excitation forces and the body motions for the very long waves can also be observed from the figures. Note that the phase shift in the surge and pitch directions of the force and motions are reversed from the head-seas to the following-seas condition.

It is important to mention that relative to the head seas, the pseudo-impulsive scattering forces in following-seas are non-trivial over a larger span of the simulation time. This can be observed by comparing the time-domain forces in Figures 25 and 32-34. This implies that for the following-seas situation, a longer computational time is required to make sure that the scattering forces have nearly died out by the end of the simulation. However this can be avoided by the asymptotic extrapolation of the tail of the force signal as has been mentioned earlier.

\section{Conclusions}

A model has been developed to solve the first-order diffraction problem with forward speed. It is based on a 4th-order finite-difference discretization method on body-fitted overlapping grids. Both the Neumann-Kelvin and the double-body linearizations have been implemented, and the incident wave can have any arbitrary heading including the following seas case. The body boundary condition has been defined based on a pseudo-impulsive Gaussian incident wave elevation. Moreover in order to consider also the finite-depth diffraction problem, the resulting integral for the incident wave velocity has been calculated by the discrete FFT, and the convergence of this procedure has been demonstrated.

The presented model has been validated against solutions from both two- and three-dimensional analytical geometries, and mostly excellent agreement has been shown, especially for case of the submerged sphere. Hyperbolic grid generation is used to build the grid for the hull of a bulk carrier, and the calculated wave excitation forces and ship motions (both in head seas and following seas) compare very well with the measurements and the numerical solutions from boundary element codes.

\section{Acknowledgement}

The authors wish to acknowledge The Danish Maritime Fund (Den Danske Maritime Fond) and Orients Fond for their generous financial support of this project. The experimental data for the RIOS bulk carrier which was provided by Prof. M. Kashiwagi from the Department of Naval Architecture \& Ocean Engineering Osaka University, and also by Prof. H. Iwashita, is greatly appreciated. The authors are also very grateful to Prof. William D. Henshaw at the Department of Mathematical Sciences, Rensselaer Polytechnic Institute, for his help regarding the Overture library, and hyperbolic grid generation for ship hulls. 


\section{References}

[1] F. Stern, J. Yang, Z. Wang, H. Sadat-Hosseini, M. Mousaviraad, S. Bhushan, and T. Xing, "Computational ship hydrodynamics: Nowadays and way forward," International Shipbuilding Progress, vol. 60, no. 1-4, pp. 3-105, 2013.

[2] H. Sadat-Hosseini, P.-C. Wu, P. M. Carrica, H. Kim, Y. Toda, and F. Stern, "CFD verification and validation of added resistance and motions of kvlcc2 with fixed and free surge in short and long head waves," Ocean Engineering, vol. 59, pp. 240-273, 2013.

[3] L. Larsson, F. Stern, and M. Visonneau, "Numerical ship hydrodynamics," in Gothenburg 2010 a Workshop on Numerical Ship Hydrodynamics, Gothenburg, pp. 1-32, Springer, 2010.

[4] L. Larsson, F. Stern, and M. Visonneau, "CFD in ship hydrodynamics - results of the Gothenburg 2010 workshop," in MARINE 2011, IV International Conference on Computational Methods in Marine Engineering, pp. 237-259, Springer, 2013.

[5] J. Wackers, B. Koren, H. C. Raven, A. Van der Ploeg, A. Starke, G. Deng, P. Queutey, M. Visonneau, T. Hino, and K. Ohashi, "Free-surface viscous flow solution methods for ship hydrodynamics," Archives of Computational Methods in Engineering, vol. 18, no. 1, pp. 1-41, 2011.

[6] H. Raven, A. Van der Ploeg, A. Starke, and L. Eça, "Towards a CFD-based prediction of ship performanceprogress in predicting full-scale resistance and scale effects," Proceedings of RINACFD-2008, London, UK, 2008.

[7] N. Salvesen, E. Tuck, and O. Faltinsen, Ship motions and sea loads. Det norske Veritas, Oslo, 1971.

[8] M. Kashiwagi, "Prediction of surge and its effect on added resistance by means of the enhanced unified theory," Trans. of West-Japan Soc. Nav. Arch., vol. 89, pp. 77-89, 1995.

[9] D. Nakos and P. Sclavounos, "Ship motions by a three-dimensional Rankine panel method," in 18th Symposium on Naval Hydrodynamics, Ann Arbor, Michigan, pp. 21-39, 1990.

[10] D. Nakos, D. Kring, and P. Sclavounos, "Rankine panel methods for transient free-surface floes," in 6th International Conference on Numerical Ship Hydrodynamics, Iowa City, Iowa, 1993, 1993.

[11] D. C. Kring, Time domain ship motions by a three-dimensional Rankine panel method. PhD thesis, Massachusetts Institute of Technology, 1994.

[12] Y. Kim, K.-H. Kim, J.-H. Kim, T. Kim, M.-G. Seo, and Y. Kim, "Time-domain analysis of nonlinear motion responses and structural loads on ships and offshore structures: development of WISH programs," International Journal of Naval Architecture and Ocean Engineering, vol. 3, no. 1 , pp. $37-52,2011$.

[13] H. Raven, "Nonlinear ship wave calculations using the rapid method," in Proc. 6th International Conference on Numerical Ship Hydrodynamics, Iowa City, Iowa, pp. 95-118, 1993.

[14] Y.-L. Shao and O. M. Faltinsen, "Linear seakeeping and added resistance analysis by means of body-fixed coordinate system," Journal of marine science and technology, vol. 17, no. 4, pp. 493$510,2012$.

[15] R. F. Beck and B. King, "Time-domain analysis of wave exciting forces on floating bodies at zero forward speed," Applied Ocean Research, vol. 11, no. 1, pp. 19-25, 1989.

[16] H. B. Bingham, Simulating ship motions in the time domain. PhD thesis, Massachusetts Institute of Technology, Department of Ocean Engineering, 1994. 
[17] F. T. Korsmeyer and H. Bingham, "The forward speed diffraction problem," Journal of ship research, vol. 42, no. 2, pp. 99-112, 1998.

[18] F. Kara and D. Vassalos, "Time domain prediction of steady and unsteady marine hydrodynamics problem," International shipbuilding progress, vol. 50, no. 4, pp. 317-332, 2003.

[19] R. Taylor Eatock, "Analysis of non-linear wave-body interactions using finite elements," in Waves and Nonlinear Processes in Hydrodynamics, pp. 51-62, Springer, 1996.

[20] G. Wu and R. Eatock Taylor, "The coupled finite element and boundary element analysis of nonlinear interactions between waves and bodies," Ocean Engineering, vol. 30, no. 3, pp. 387400, 2003.

[21] C. Dawson, "A practical computer method for solving ship-wave problems," in Proceedings of Second International Conference on Numerical Ship Hydrodynamics, pp. 30-38, 1977.

[22] H. Miyata, S. Nishimura, and A. Masuko, "Finite difference simulation of nonlinear waves generated by ships of arbitrary three-dimensional configuration," Journal of Computational Physics, vol. 60, no. 3, pp. 391-436, 1985.

[23] H. B. Bingham and H. Zhang, "On the accuracy of finite-difference solutions for nonlinear water waves," Journal of Engineering Mathematics, vol. 58, no. 1-4, pp. 211-228, 2007.

[24] A. P. Engsig-Karup, O. Lindberg, S. L. Glimberg, B. Dammann, H. B. Bingham, and P. A. Madsen, "A high-order WENO finite difference water wave model for interactive ship-wave simulation," in International Conference on Spectral and High Order Methods (ICOSAHOM 2012), 2012 .

[25] S. Kontos, H. B. Bingham, O. Lindberg, and A. P. Engsig-Karup, "A robust WENO scheme for nonlinear waves in a moving reference frame," Journal of Hydrodynamics, Ser. B, vol. 28, no. 3, pp. 482-488, 2016.

[26] M. Amini-Afshar and H. B. Bingham, "Solving the linearized forward-speed radiation problem using a high-order finite difference method on overlapping grids," Applied Ocean Research, vol. 69, pp. 220-244, 2017.

[27] M. Amini-Afshar, H. B. Bingham, and R. Read, "A high-order finite-difference linear seakeeping solver tool for calculation of added resistance in waves," in 30th International Workshop on Water Waves and Floating Bodies, (Bristol, UK), 2015.

[28] M. Amini-Afshar, H. B. Bingham, and R. Read, "A high-order finite-difference solver for the linearised potential flow wave resistance problem on curvilinear overset grids," in 30th International Workshop on Water Waves and Floating Bodies, (Osaka, Japan), 2014.

[29] A. P. Engsig-Karup, M. G. Madsen, and S. L. Glimberg, "A massively parallel GPU-accelerated model for analysis of fully nonlinear free surface waves," International Journal for Numerical Methods in Fluids, vol. 70, no. 1, pp. 20-36, 2012.

[30] R. Read and H. B. Bingham, "Solving the linear radiation problem using a volume method on an overset grid," in 27th International Workshop on Water Waves and Floating Bodies, (Copenhagen, Denmark), 2012.

[31] M. Amini-Afshar, H. B. Bingham, W. Henshaw, and R. Read, "Convergence of near-field added resistance calculations using a high-order finite-difference method," in Proceedings of 13th PRADS, 2016 . 
[32] R. J. LeVeque, Finite difference methods for ordinary and partial differential equations: steadystate and time-dependent problems, vol. 98. Siam, 2007.

[33] B. King, "Time-domain analysis of wave exciting forces on ships and bodies," tech. rep., University of Michigan, 1987.

[34] M. Amini-Afshar, H. B. Bingham, and R. Read, "OceanWave3D-Seakeeping." https://gitlab.gbar.dtu.dk/oceanwave3d/ow3d-seakeeping.git, 2015.

[35] J. Newman, "The theory of ship motions," Advances in Applied Mechanics, vol. 18, pp. 221-283, 1979 .

[36] D. L. Brown, W. D. Henshaw, and D. J. Quinlan, "Overture: An object-oriented framework for solving partial differential equations on overlapping grids," Object Oriented Methods for Interoperable Scientific and Engineering Computing, SIAM, pp. 245-255, 1999.

[37] G. Wu, "Hydrodynamic forces on a submerged cylinder advancing in water waves of finite depth," Journal of Fluid Mechanics, vol. 224, pp. 645-659, 1991.

[38] G. Wu and R. Eatock Taylor, "Radiation and diffraction of water waves by a submerged sphere at forward speed," Proceedings of the Royal Society of London. A. Mathematical and Physical Sciences, vol. 417, no. 1853, pp. 433-461, 1988.

[39] W. M. Chan, "Hyperbolic methods for surface and field grid generation," in Handbook of Grid Generation (S. Thompson and Weatherill, eds.), ch. 5, pp. 5-1-5-26, CRC Press, 1998.

[40] W. D. Henshaw, "Ogen: An overlapping grid generator for overture," LANL unclassified report, pp. 96-3466, 1998.

[41] H. Iwashita, M. Kashiwagi, Y. Ito, and Y. Seki, "Calculations of ship seakeeping in low-speed/lowfrequency range by frequency-domain Rankine panel methods," Journal of the Japan Society of Naval Architects and Ocean Engineers, vol. 24, pp. 129-146, 2016, (In Japanese).

[42] F. Korsmeyer, H. Bingham, and J. Newman, TiMIT - A Panel Method for Transient Wave-Body Interactions. Research Laboratory of Electronics, MIT, 1999. 